\title{
Retroactive Packet Sampling for Traffic Receipts
}

\author{
PAVLOS NIKOLOPOULOS*, CHRISTOS PAPPAS ${ }^{+}$, KATERINA ARGYRAKI*, ADRIAN \\ PERRIG ${ }^{+},{ }^{*}$ EPFL, Switzerland, ${ }^{+}$ETHZ, Switzerland
}

Is it possible to design a packet-sampling algorithm that prevents the network node that performs the sampling from treating the sampled packets preferentially? We study this problem in the context of designing a "network transparency" system. In this system, networks emit receipts for a small sample of the packets they observe, and a monitor collects these receipts to estimate each network's loss and delay performance. Sampling is a good building block for this system, because it enables a solution that is flexible and combines low resource cost with quantifiable accuracy. The challenge is cheating resistance: when a network's performance is assessed based on the conditions experienced by a small traffic sample, the network has a strong incentive to treat the sampled packets better than the rest. We contribute a sampling algorithm that is provably robust to such prioritization attacks, enables network performance estimation with quantifiable accuracy, and requires minimal resources. We confirm our analysis using real traffic traces.

\section{CCS Concepts: • Networks $\rightarrow$ Data path algorithms; Network measurement;}

ACM Reference Format:

Pavlos Nikolopoulos*, Christos Pappas ${ }^{+}$, Katerina Argyraki*, Adrian Perrig ${ }^{+}$. 2019. Retroactive Packet Sampling for Traffic Receipts. Proc. ACM Meas. Anal. Comput. Syst. 3, 1, Article 19 (March 2019), 39 pages. https://doi.org/10.1145/3311090

\section{INTRODUCTION}

We study the following problem: is it possible to design a packet-sampling algorithm that prevents the network node that performs the sampling from treating the sampled packets preferentially? For instance, consider the Internet Service Provider (ISP) shown in Fig. 1 and suppose that it emits "traffic receipts" (essentially digests and timestamps) for a small sample of the packets that enter and exit its network; a monitor collects these receipts and uses them to periodically estimate the ISP's loss rate and delay distribution. Since the ISP's performance is estimated based on how it treats the sampled packets, the ISP has an incentive to cheat: treat the sampled packets better than the rest (e.g., forward them through a lower-loss, lower-delay intra-domain path) in order to exaggerate its performance. Is it possible to design a packet-sampling algorithm that prevents such behavior?

Our motivation for studying this problem is the need for more network transparency. In the current Internet, when packets get lost or delayed, there is typically no information about where the problem occurred, hence no information about who is responsible. This results in service level agreements (SLAs) and neutrality regulations that cannot be enforced: First, ISPs guarantee that their network will honor a minimum delivery rate (equivalently, a maximum loss rate) and a maximum latency $[8,9,23]$, even though there exists no systematic way to estimate their loss rate or delay distribution. Second, governments require that ISPs not (de)prioritize certain traffic classes $[7,10,20]$, even though there exists no systematic way to detect traffic (de)prioritization. We

Author's address: Pavlos Nikolopoulos*, Christos Pappas ${ }^{+}$, Katerina Argyraki* ${ }^{*}$ Adrian Perrig ${ }^{+*}$ EPFL, Switzerland, ${ }^{+}$ETHZ, Switzerland.

Permission to make digital or hard copies of all or part of this work for personal or classroom use is granted without fee provided that copies are not made or distributed for profit or commercial advantage and that copies bear this notice and the full citation on the first page. Copyrights for components of this work owned by others than ACM must be honored. Abstracting with credit is permitted. To copy otherwise, or republish, to post on servers or to redistribute to lists, requires prior specific permission and/or a fee. Request permissions from permissions@acm.org.

(c) 2019 Association for Computing Machinery.

2476-1249/2019/3-ART19 \$15.00

https://doi.org/10.1145/3311090

Proc. ACM Meas. Anal. Comput. Syst., Vol. 3, No. 1, Article 19. Publication date: March 2019. 


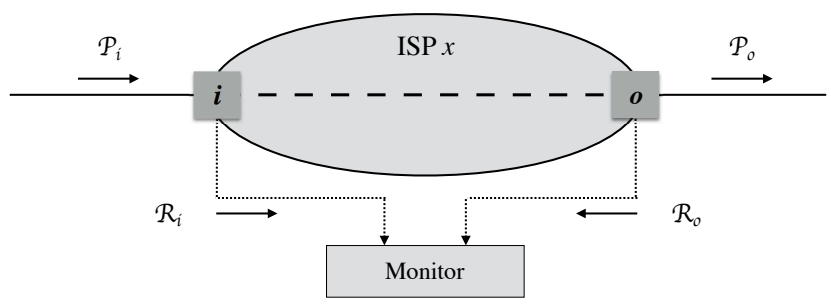

Fig. 1. Example.

$\mathcal{P}_{i}$ and $\mathcal{P}_{o}$ are the packet streams observed, respectively, by nodes $i$ and $o$. $\mathcal{R}_{i}$ and $\mathcal{R}_{o}$ are the receipts emitted, respectively, by nodes $i$ and $o$.

are not arguing in favor of SLAs or neutrality regulations; just that, if network users care enough for them to exist, then there should be a way to check whether they are honored.

We support the idea of a "network-transparency system" [1-4, 19, 21, 24-26], where each participating network emits receipts for traffic it receives and delivers. The receipts are crafted such that an independent monitor can process them and estimate the network's mean loss rate and delay distribution quantiles with respect to various traffic aggregates. Hence, the monitor can check whether the network honors SLAs and/or neutrality regulations. Networks participate either because they are expected to by their governments, or (our preferred scenario) by their own choice, because producing verifiable evidence of honoring SLAs and neutrality regulations is good for their reputation. Today, even if an ISP wants to produce such verifiable evidence, e.g., to defend itself against wrongful suspicion of SLA or neutrality violation, it has no way of doing it.

Where we differ from most prior work is in the nature of the traffic receipts: we argue that each participating network should emit receipts for a small sample of the packets that it receives and delivers. Two factors led us to sampling: (a) The need for flexibility: The monitor should be able to estimate network performance with respect to any packet aggregate, precluding solutions that emit "summary receipts" for specific, pre-determined packet aggregates. (b) The need to balance resource cost and accuracy: A network should compute the necessary receipts and export them to the monitor without a significant increase in equipment cost, precluding solutions that require emitting per-packet receipts. At the same time, a network should emit enough receipts for the monitor to estimate the network's performance with a desired accuracy in a desired time interval. Sampling is a good starting point, because it enables balancing these two goals by tuning the sampling probability.

The challenge is resistance to misbehavior: a network that participates in a transparency system should not be able to misbehave and exaggerate its performance, and we want to meet this goal without trusted hardware. Prior work on fault localization [1-4, 14, 24-26] has solved one aspect of the challenge, which is how to deal with fake reports, e.g., a network reporting that it delivered certain traffic whereas it actually dropped it. We focus on another aspect that remains unsolved, which is how to deal with "prioritization attacks": as stated above, when a network's performance is estimated based on how it treats a small sample of forwarded packets, the network has an incentive to bias the sample-treat the sampled packets better than the rest-resulting in arbitrarily inaccurate estimation of its performance.

Of all the technical challenges in designing and building a network transparency system, and of all the possible misbehavior, why focus on prioritization attacks? Packet sampling is among the most popular and well-studied concepts in networking, both among practitioners and researchers, because it enables the computation of flexible statistics with good, configurable accuracy and at low, configurable resource cost. Our vision is to leverage this popularity and build a system where the 
data-plane of each network emits information on a small, configurable sample of observed traffic, and this information is used both for the network's internal management and troubleshooting (as already happens today) and (after proper filtering) for network transparency. For this to work, the fundamental challenge is ensuring that the sample is representative without assuming that the data-plane, which chooses and handles the sample, is trusted; this is what we set out to solve.

We build on the idea of sampling with delayed disclosure [3]: when a network node observes a packet $p$, it cannot immediately determine whether it should sample $p$ or not, because that information is disclosed by subsequent traffic; by the time disclosure happens, the node has normally forwarded $p$, hence cannot treat it according to its sampling fate. We call the sampling algorithm proposed in [3] "basic delayed disclosure."

Basic delayed disclosure is promising, however, an analysis of the algorithm reveals flaws: First, vulnerability to subtle prioritization attacks (§7.4), where a misbehaving network buffers packets long enough to learn their sampling fate with a non-trivial probability, yet short enough not to introduce significant buffering delays; we show that such an attack enables a misbehaving network to claim significantly less loss and delay (as much as $41 \%$ in our experiments) than it actually introduces. Second, in many realistic scenarios, achieving good accuracy in a timely manner requires many tens of MBs of data-path memory per $10 \mathrm{Gbps}$ of forwarding capacity (§6); at this cost, we might as well use a completely different approach from sampling, e.g., maintain explicit per-flow loss and delay information on the data-path, which is not vulnerable to prioritization attacks in the first place.

Our contribution is a sampling algorithm (§3) that corrects these flaws: First, it is robust to prioritization attacks ( $(4)$. Second, it uses the minimum amount of data-path memory necessary for achieving a desired accuracy in a desired time interval (§5). As a result, it achieves good accuracy in a timely manner, while requiring a modest amount of resources, affordable by modern networks; for instance, in the same scenario where basic delayed disclosure requires many tens of MBs of data-path memory per $10 \mathrm{Gbps}$ of forwarding capacity, our algorithm requires only a couple of $\mathrm{MB}-$ an order of magnitude less (§6). To achieve these properties, we enhance delayed disclosure with carefully regulated "quiet periods," during which no sampling may occur, and with a new disclosure process, which is continuously adapted to the observed traffic. These two techniques allow us to control the pace of disclosure, such that we emit no more receipts than necessary and make prioritization attacks ineffective: by the time a misbehaving network has learned the sampling fate of a packet (with a non-trivial probability), it has buffered-hence delayed-the packet for so long that it cannot benefit from prioritization any more. Our experimental evaluation confirms our analysis using real traffic traces $(§ 7)$.

\section{SETUP}

In this section, we state our terminology (§2.1), the problem we solve (2.2), the starting point for our solution (§2.3), our trust model (§2.4), and our assumptions and limitations (§2.5).

\subsection{Terminology}

A "domain" is a contiguous network area managed by a single administrative entity, e.g., an ISP, an Autonomous System (AS), an Internet eXchange Point (IXP), an enterprise or campus network, the data-center network of a content provider.

Each domain that participates in our system deploys a special "node" at each point where it exchanges traffic with another domain. Each node runs an algorithm (from now on referred to as "the algorithm") that takes as input a set of configuration parameters and the sequence of packets 
arriving at the node, and outputs a set of "receipts." A node is always collocated with a border router and can be implemented on the linecards that handle the packets as they enter and exit the domain.

The "monitor" is an entity that collects the receipts emitted by the participating domains and uses them to estimate domain performance. It can be owned and managed by one authority, e.g., like the root DNS servers, or it can be a decentralized system, owned and managed by the participating domains themselves.

We define two kinds of traffic units: "flows" and "aggregates." A "flow" is the set of packets observed by a node that have the same source and destination IP prefix. An "aggregate" is a set of packets with some common observable characteristic, e.g., the set of packets from a given source to a given destination domain, or all BitTorrent packets from a given source domain; so, an aggregate may be a flow's subset or consist of one or multiple flows. An aggregate's "source node" is the first node that observes all traffic from that aggregate. Nodes can classify packets per flow but are not aware of aggregates. The monitor, on the other hand, estimates domain performance with respect to aggregates that it defines as needed (examples in §7.2).

In our context, the monitor is trusted, while a node (and the domain that owns the node) may "misbehave," i.e., try to cause the monitor to produce incorrect estimates that exaggerate the domain's performance.

\subsection{Goals}

We set three goals:

1) Given an aggregate $A$, the monitor should be able to estimate each participating domain's mean loss rate and delay distribution quantiles with respect to $A$ with a given target $(\gamma, \epsilon)$-accuracy, where $\gamma$ is the probability that the relative estimation error is below $\epsilon$. This is a standard accuracy metric for loss estimates and was recently defined for delay estimates as well [5, 22].

2) A domain should be able to deploy and run the algorithm on a node without increasing the node's data-to-control-path bandwidth and data-path memory by more than a few percentage points.

3) A misbehaving domain should not be able to significantly exaggerate its performance by manipulating either the receipts emitted by its nodes or their forwarding process.

\subsection{Starting Point: Sampling}

Each node emits a receipt for a small sample of packets, drawn from all the packets it observes. Each receipt carries: a digest that uniquely identifies the packet with high probability; a timestamp that specifies when the packet was observed at the node; and a flow ID that specifies the packet's source and destination IP prefix.

Each node draws the sample it reports on from all the packets it observes. If, instead, we reactively configured the nodes to emit receipts for a specific aggregate of interest $A$, e.g., in reaction to user suspicions that $A$ is being throttled, then a dishonest domain could change how it treats $A$ the moment it starts reporting on it.

\subsection{Attack Model}

We classify misbehavior into "fake receipts," where a domain emits incorrect receipts that exaggerate its performance; and "prioritization," where a domain emits correct receipts but manipulates its forwarding process in order to exaggerate its performance. We briefly discuss the former here and focus on the latter in the rest of the paper. 
In a fake-receipt attack, a node emits a set of receipts that is different from the one produced by the algorithm. For example, it may suppress a receipt (pretend that it never received a packet that it actually did receive and dropped), emit a superfluous receipt (pretend that it delivered a packet that it actually dropped), or modify receipt timestamps (pretend that it received a packet later, or delivered a packet earlier than it actually did).

We now outline how these attacks can be addressed based on ideas from prior work $[1-4,14$, 24-26]. One way to prevent fake-receipt attacks is through incentives: (1) If domain $x$ 's receipt manipulation does not improve $x$ 's estimated performance, then $x$ has no incentive to launch such an attack. (2) If $x$ 's receipt manipulation necessarily and visibly implicates $x$ 's neighbor $y$, then $x$ has an incentive to not launch such an attack, as that would affect its business relationship to $y$. Such incentives can be created with consistent sampling [27]: Each node hashes part of the non-mutable contents of each observed packet and samples the packet if the outcome falls within a pre-determined range $[13,18]$. A hash function with strong randomization properties results in uniform sampling, where the sampling probability is determined by the range of the hash function As a result, a packet is either sampled by all the nodes that observe it or by none of them.

Definition 2.1. Consider a packet $p$ from a flow $F$ that traverses a node $i$ and then another node $o$. We say that nodes $i$ and $o$ "sample $p$ consistently" if the conditional probability that $o$ samples $p$ given that $o$ observes $p$ and $i$ samples $p$ is 1 .

With consistent sampling, a fake receipt shifts the blame for a lost or delayed packet to an inter-domain link; since an inter-domain link is shared responsibility with a neighbor, a fake receipt does not exonerate the culprit, while it necessarily and visibly implicates the culprit's neighbor. For example, suppose domain $y$ delivers packet $p$ to domain $x$, which drops it; both $y$ and $x$ sample $p$, but $x$ suppresses the receipt produced for $p$ at its entry point, i.e., pretends that it never received $p$. Since $y$ produces a receipt for $p$ but $x$ does not, the monitor concludes that $p$ was dropped on the inter-domain link between $y$ and $x$, and it attributes the loss to both $x$ and $y$. Hence, $x$ is not exonerated, and it also implicates $y$. Moreover, $y$ can monitor its inter-domain link with $x$, determine that it is not faulty/congested (hence cannot have dropped $p$ ), and detect $x$ 's misbehavior.

In a prioritization attack, a node emits the set of receipts that is produced by the algorithm but manipulates its forwarding process: it treats some or all of the sampled packets preferentially, e.g., by assigning them to higher-priority/lower-priority queues or routing them through better/worse paths. Prior work has considered these attacks [3] but not solved them (§7.4).

\subsection{Assumptions and Limitations}

The monitor employs standard statistical techniques to compute loss and delay estimates; to provide confidence intervals, these techniques must assume something about the nature of the loss that is being estimated, and the typical assumption is that loss is either i.i.d. (independent identically distributed) across all packets or follows the Gilbert [16] model.

We do not assume independent/Poisson packet arrivals. Our analysis assumes that:

(1) Each flow F's packet arrivals (resp. all packet arrivals) at each node form a stationary, ergodic point process with intensity $r$ (resp. $R$, generally different for each node), which is in its stationary regime.

(2) Each flow F's intensity is high enough (e.g., corresponds to the packet rate of a highly utilized OC-12 link or higher) that ergodic convergence is achieved in less than $100 \mathrm{msec}$.

The limitation resulting from these assumptions is that we cannot reason about aggregates that consist of relatively few packets, e.g., an aggregate that consists of a typical TCP flow. We think 


\begin{tabular}{|c|c|}
\hline \multicolumn{2}{|r|}{ Symbols } \\
\hline$p$ & A packet \\
\hline$d$ & A disclosure packet \\
\hline$F$ & A flow \\
\hline \multicolumn{2}{|r|}{ Workload characteristics } \\
\hline$r$ & Intensity (in packets/sec) of a flow's packet arrivals at a node \\
\hline$R$ & Intensity (in packets/sec) of all packet arrivals at a node \\
\hline \multicolumn{2}{|r|}{ Algorithm parameters } \\
\hline$\beta$ & The size of the receipt buffer (different at each node) \\
\hline$\kappa$ & The duration of the quiet period \\
\hline$\mu$ & Jitter margin: subset of the quiet period during which packets are sampled \\
\hline$\delta_{r}$ & $\begin{array}{l}\text { Disclosure rate: probability that a node picks a packet from a flow } \\
\text { with intensity } r \text { as a disclosure packet }\end{array}$ \\
\hline$\sigma$ & $\begin{array}{l}\text { Selection rate: conditional probability that a node samples a packet } \\
\text { given that the packet does not suffer early or late disclosure at the node }\end{array}$ \\
\hline \multicolumn{2}{|r|}{ Other configuration parameters } \\
\hline$(\gamma, \epsilon)$ & Target accuracy (confidence level and error) for the monitor's estimates \\
\hline$T$ & Target time interval in which the target accuracy should be achieved \\
\hline$N_{\gamma, \epsilon}$ & Minimum number of samples needed to achieve the target accuracy \\
\hline
\end{tabular}

Table 1. Parameters and symbols.

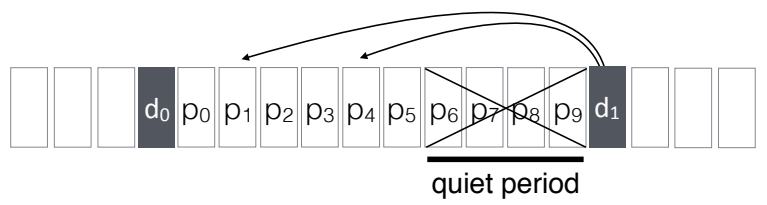

Fig. 2. Delayed disclosure.

that this limitation is acceptable given our motivation to enable the verification and enforcement of SLAs or neutrality regulations, which typically apply to relatively large aggregates.

\section{ALGORITHM}

In this section, we describe our algorithm, which is called "retroactive packet sampling." We first describe the parts adopted from prior work (§3.1), then the novel parts $(\S 3.2,3.3)$ and the rationale behind them (§3.4). We also summarize how the monitor estimates performance based on the algorithm’s output (§3.5). Table 1 states all the relevant symbols; we use greek letters for algorithm parameters and latin letters for other symbols.

\subsection{Basic Delayed Disclosure}

We adopted the following idea from prior work [3]: at each node, certain packets, called "disclosure packets," determine the sampling fate of the previously observed packets from the same flow. For example, Fig. 2 shows a sequence of packets from a flow $F$ observed at a node; $d_{1}$ is a disclosure packet and determines that, among previously observed packets $p_{0} \ldots p_{9}$, only $p_{1}$ and $p_{4}$ will be sampled.

In more detail: The algorithm (Alg.1) takes as input the sequence of packets arriving at the local node, and it outputs receipts for a sample of these packets, which are then exported to the monitor. The node maintains a circular "receipt buffer" of size $\beta$ and adds, at the tail of this buffer, a receipt for every new packet (lines 1,2). When a new packet $d$ arrives, the node picks $d$ as a disclosure packet with some probability, by applying a hash function with strong randomization properties 


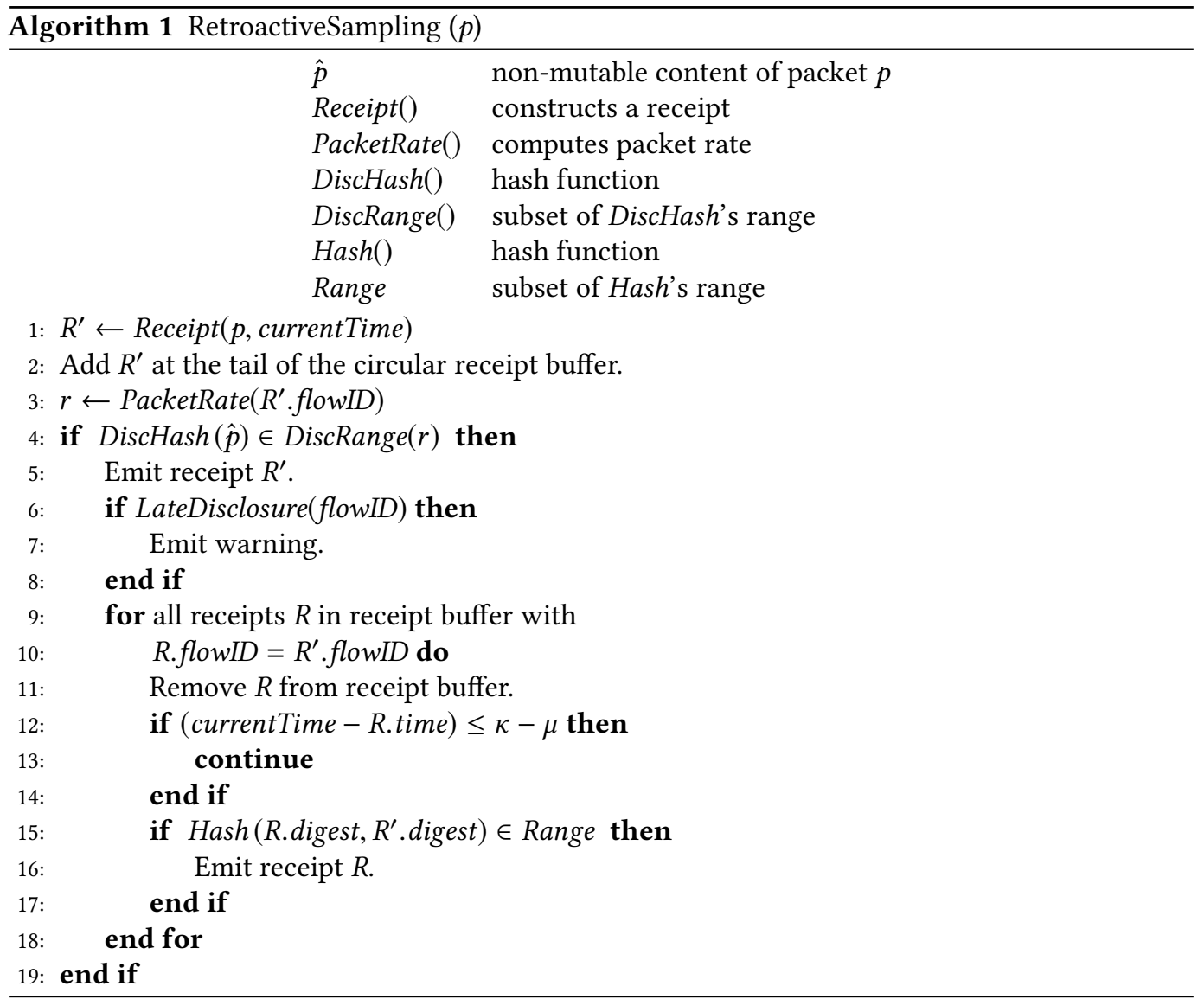

on $d$ 's non-mutable content (line 4). If $d$ is picked as a disclosure packet, the node samples $d$ (line 5), removes from the receipt buffer all receipts with the same flowID as $d$ (lines 9-11), and picks some of them for sampling (lines 15, 16).

There are two important points about disclosure packets: (1) Whether a node samples a packet $p$ or not depends on the next disclosure packet $d$ from the same flow as $p$ that arrives at the node; we say that $d$ is " $p$ 's disclosure packet," and that "disclosure for $p$ happens" when $d$ arrives at the node. (2) Disclosure packets are normal packets that happen to be picked by the nodes to play a particular role in the sampling algorithm. Disclosure packets are not explicitly labeled by anyone, nor explicitly introduced into the traffic stream by the monitor, the nodes, or the traffic sources. If two nodes observe the same flow, they mostly pick the same packets as disclosure packets, by virtue of using the same function on line 4 .

\subsection{Late Disclosure}

We say that a packet $p$ "suffers late disclosure" at a node, when $p$ 's disclosure packet arrives at the node after $p$ 's receipt in the node's circular receipt buffer has been overwritten (in which case, lines 9 to 18 are never executed for $p$ ).

When a node picks a new disclosure packet, it checks for late disclosure and warns the monitor. More specifically, when a node picks a packet from a flow $F$ as a disclosure packet, it checks whether the receipt for the previous disclosure packet from $F$ is still in the buffer (line 6); if yes, it is certain 
that none of $F$ 's packets that arrived between the two disclosure packets suffered late disclosure; if not, the node emits a "late disclosure" warning (line 7), referencing the earliest of $F$ 's packets whose receipt is still in the buffer.

For example, in Fig. 2, a node receives packets $d_{0}, p_{0}, \ldots p_{9}, d_{1}$ from a flow $F$ and picks $d_{0}$ and $d_{1}$ as disclosure packets; hence, $d_{1}$ is the disclosure packet for $p_{0} \ldots p_{9}$. Suppose that $p_{0}$ and $p_{1}$ suffer late disclosure at the node: by the time $d_{1}$ arrives, the receipts for $d_{0}, p_{0}$, and $p_{1}$ have been overwritten. When the node picks $d_{1}$ as a disclosure packet, it detects that $d_{0}$ 's receipt is not in the buffer, and also that $p_{2}$ is the earliest packet from $F$ whose receipt is still in the buffer; hence, the node emits a late-disclosure warning referencing $p_{2}$, which signals to the monitor that packets from flow $F$ that arrived at the node between $d_{0}$ and $p_{2}$ suffered late disclosure.

\subsection{Quiet Periods and Adaptive Disclosure}

We want disclosure to happen too late to be useful to a misbehaving node/domain; the challenge lies in achieving this goal for all the flows observed by each node without violating the simplicity of basic delayed disclosure.

First, we impose a "quiet period" of duration $\kappa$ before each disclosure packet: a node does not sample any packet $p$ that arrives within $\kappa-\mu$ from its disclosure packet (lines 12,13), where $\mu$ is a "jitter margin" used to help consistent sampling (§3.5). We say that a packet $p$ "suffers early disclosure" at a node, when $p$ arrives at the node during a quiet period. For example, in Fig. 2, packets $p_{6}$ to $p_{9}$ arrive during the quiet period that precedes their disclosure packet $d_{1}$, hence suffer early disclosure.

Second, we adapt the disclosure process to each flow's intensity: When a new packet $p$ from a flow $F$ arrives at a node, the node roughly estimates $F$ 's intensity $r$ (line 3 ) and picks $p$ as a disclosure packet with a probability $\delta_{r}$ that depends on $r$. In the Appendix, Section E.2, we describe a hardware-friendly implementation that tracks flow intensities without maintaining explicit per-flow state.

When disclosure for a packet $p$ happens at a node neither late nor early, the node samples $p$ with a fixed probability $\sigma$, by applying a hash function with strong randomization properties on $p$ 's and its disclosure packet's non-mutable contents (lines 15, 16). For example, in Fig. 2, after picking $d_{1}$ as a disclosure packet, the node samples previously observed packets $p_{1}$ and $p_{4}$.

\subsection{Rationale}

Our algorithm is the result of combining delayed disclosure with consistent sampling: The former requires that each packet $p$ 's sampling fate be determined by a subsequent disclosure packet; the latter requires that all nodes that observe $p$ make the same sampling decision for it. To satisfy both requirements, all nodes that observe $p$ should pick the same disclosure packet for it, which means that $p$ and its disclosure packet should traverse the same nodes. In the current Internet architecture, there is no way to guarantee this; the best indication a node has that two arriving packets have traversed and will continue to traverse the same nodes is that they share the same flow-the same source and destination IP prefix-which is why our algorithm always picks $p$ 's disclosure packet from the same flow as $p$. A side-effect of this design choice is that nodes occasionally sample inconsistently, e.g., due to loss, reordering, or rerouting of disclosure packets. However, in most cases, the monitor can identify and discard receipts for inconsistently sampled packets (§3.5).

Quiet periods make prioritization attacks more expensive by ensuring that the decision to sample a packet $p$ is never made within time $\kappa$ from $p$ 's observation. By tuning $\kappa$, we can control how much a misbehaving domain worsens its perceived delay performance (§4.2). 
The adaptation of the disclosure process to each flow's intensity regulates early and late disclosure and ensures that each node produces enough receipts from each flow to enable accurate statistics. Both early and late disclosure exclude packets from sampling; hence, if we want a node to sample packets from a flow $F$ at some minimum rate, we have to ensure that enough packets from $F$ escape early and late disclosure. The probability of early disclosure depends on the interplay between $F$ 's intensity $r$, the disclosure rate $\delta$, and the quiet-period duration $\kappa$; this interplay determines how many of $F$ 's packets fall into a quiet period. The probability of late disclosure depends on the interplay between $r, \delta$, the rest of the traffic arriving at the node, and the size of the receipt buffer $\beta$; this interplay determines how quickly the receipt buffer fills up and how many of $F$ 's receipts are overwritten prematurely. So, to keep the probabilities of early and late disclosure at a desired value, we have to quantify the above interactions and continuously adapt the disclosure process to $F$ 's intensity-this is why nodes track flow intensities and why $\delta$ is a function of $r$.

\subsection{Performance Estimation}

Consider an aggregate $A$ that traverses a node $i$ and then another node $o$. The monitor estimates the performance experienced by $A$ between $i$ and $o$ during time interval $T$ in a straightforward manner:

(1) Collects all the receipts $\mathcal{R}_{i}$, emitted by $i$, and $\mathcal{R}_{o}$, emitted by $o$, during $T$.

(2) Identifies which receipts belong to $A$ 's packets.

(3) Identifies and removes from $\mathcal{R}_{i}$ and $\mathcal{R}_{o}$ any receipts for packets that suffered early disclosure at either $i$ or both nodes, or were sampled inconsistently by $i$ and $o$.

(4) Estimates mean loss rate as $\frac{\left|\mathcal{R}_{i}\right|-\left|\mathcal{R}_{o}\right|}{\left|\mathcal{R}_{i}\right|}$.

(5) Estimates the delay distribution as $\left\{R_{o}\right.$.time $-R_{i}$.time $\mid R_{o}$.digest $=R_{i}$.digest $\}$, for all $R_{o} \in \mathcal{R}_{o}, R_{i} \in \mathcal{R}_{i}$.

Step (2) depends on $A$ 's definition. For instance, if $A$ is defined as all packets from a flow, the monitor identifies which receipts belong to $A$ 's packets by looking at their flowID. But if $A$ is defined as a subset of a flow $F$, the monitor needs help from $F$ 's source node to identify which of $F$ 's receipts belong to $A$.

In Step (3), the monitor detects when nodes $i$ and $o$ sample inconsistently and discards the corresponding receipts. For instance, suppose $i$ observes packets $p$ and $d$, picks $d$ as $p$ 's disclosure packet, and samples $p$; suppose $o$ also observes $p$. According to Alg. 1, $o$ will also sample $p$ unless one of the following occurs:

I. Node $o$ picks a different disclosure packet for $p$ because:

(a) $d$ does not traverse $o$ due to loss or routing; or

(b) $d$ arrives at $o$ before $p$ due to reordering.

II. Node $o$ picks $d$ as $p$ 's disclosure packet but $p$ suffers early disclosure at $o$.

III. Node $o$ picks $d$ as $p$ 's disclosure packet but $p$ suffers late disclosure at $o$.

In Case I(a), the monitor knows what happened because $o$ does not emit a receipt for $d$. In Case I(b), the monitor detects with a high probability that $d$ experienced reordering between $i$ and $o$ because $o$ emits receipts for packets that $i$ does not emit receipts for. In Case II, the monitor's behavior depends on the difference, $\Delta$, between $p$ 's and $d$ 's arrival at $o$ : If $\Delta>\kappa-\mu$, then $o$ emits a receipt for $p$ despite the early disclosure (Alg. 1, line 12). If $\Delta \leq \kappa-\mu$, then $o$ does not emit a receipt for $p$ (Alg. 1, line 13), and the monitor counts $p$ as lost. The latter can happen only if the jitter between nodes $i$ and $o$ exceeds $\mu$. In Case III, the monitor again knows what happened because $o$ emits a late-disclosure warning (Alg. 1, line 7). 
The accuracy of the monitor's estimates depends on the number of samples on which they are based. Hence, the monitor picks a target $(\gamma, \epsilon)$-accuracy and computes the minimum number of samples $N_{\gamma, \epsilon}$ needed to achieve this accuracy. For this computation, the monitor relies on standard statistical models (Appendix, §D.1), parametrized with the minimum loss rate loss $_{\min }$ that the monitor should be able to measure accurately and potentially the maximum loss burst size burst $_{\max }$. The parameter values depend on the scenario: When estimating the loss of a domain that promises maximum loss $\ell$, loss $_{\min }$ should be set to $\leq \ell$; the typical loss rate mentioned in today's SLAs is $0.1 \%[8,9,23]$, so this is the default value we use in our examples. When assuming Gilbert loss, we use burst $_{\max }=2$, because we experimentally found that this value is conservative even for highly congested environments.

\section{MISBEHAVIOR ANALYSIS}

In this section, we study prioritization attacks: we present an attack model that captures all such attacks (§4.1) and sufficient conditions under which any prioritization attack is ineffective given the assumptions in $\S 2.5(\S 4.2)$. We discuss other misbehavior in the Appendix, §B.

\begin{tabular}{|c|c|}
\hline \multicolumn{2}{|r|}{ Attack parameters (unknown to us) } \\
\hline$\left\{l_{g}, d_{g}\right\}$ & Loss/delay of good route \\
\hline$\left\{l_{b}, d_{b}\right\}$ & Loss/delay of bad route \\
\hline$g$ & Fraction of traffic sent over good route \\
\hline$t$ & Buffering period \\
\hline \multicolumn{2}{|r|}{ Symbols used in analysis } \\
\hline$\left\{\hat{l}_{h o n}, \hat{d}_{h o n}\right\}$ & Loss/delay estimates if node $i$ is honest \\
\hline$\left\{\hat{l}_{m i s}, \hat{d}_{m i s}\right\}$ & Loss/delay estimates if node $i$ misbehaves \\
\hline$l_{h o n}-l_{m i s}$ & Loss benefit \\
\hline$\hat{d}_{\text {hon }}-\hat{d}_{\text {mis }}$ & Delay benefit \\
\hline$F$ & Denotes both a flow and the stationary and ergodic process of its packet arrivals at a node \\
\hline$P$ & The stationary and ergodic process of all packet arrivals at a node \\
\hline$n_{F}[0, \kappa)$ & The number of $F$-points that occur within time $\kappa$ after a packet $p$ s arrival \\
\hline$n_{F}\left[P_{0}, P_{\beta}\right)$ & The number of $F$-points that occur within $\beta P$-points after a packet $p$ s arrival \\
\hline
\end{tabular}

Table 2. Attack parameters and symbols.

\subsection{Prioritization Attack Model}

Consider a domain $x$ with an entry node $i$ and an exit node $o$, and two routes between $i$ and $o$ : a "good" route with mean loss and delay $\left\{l_{g}, d_{g}\right\}$ and a "bad" route with mean loss and delay $\left\{l_{b} \geq l_{g}, d_{b} \geq d_{g}\right\}$. Consider a flow $F$ that traverses node $i$ and then node $o$. F's packet arrivals (resp. all packet arrivals) at $i$ form a stationary, ergodic process that has intensity $r$ (resp. $R$ ).

In a prioritization attack, node $i$ delays forwarding $F$ 's packets for a maximum "buffering period" $t$; if it learns a packet $p$ 's sampling fate while $p$ is still buffered, it forwards $p$ over the good or bad route accordingly. The attack is successful when the benefit from forwarding packets based on their sampling fate outweighs the cost of buffering-hence delaying-packets.

More specifically: Node $i$ forwards a fraction $g \in[0,1)$ of $F$ 's packets over the good route and the rest over the bad route. Upon receiving a packet $p$, node $i$ runs Alg. 1, then Alg. 2: It buffers $p$ for a maximum period $t \geq 0$ (line 1). If $i$ learns that $p$ will not be sampled (lines 2-4), it forwards $p$ over the bad route if possible (lines 5,6). If $i$ learns that $p$ will be sampled (line 7), it forwards $p$ over the good route if possible (lines 8,9). Finally, if the buffering period runs out before disclosure occurs, then $i$ forwards $p$ over the good or the bad route (line 13) based on an arbitrary forwarding 


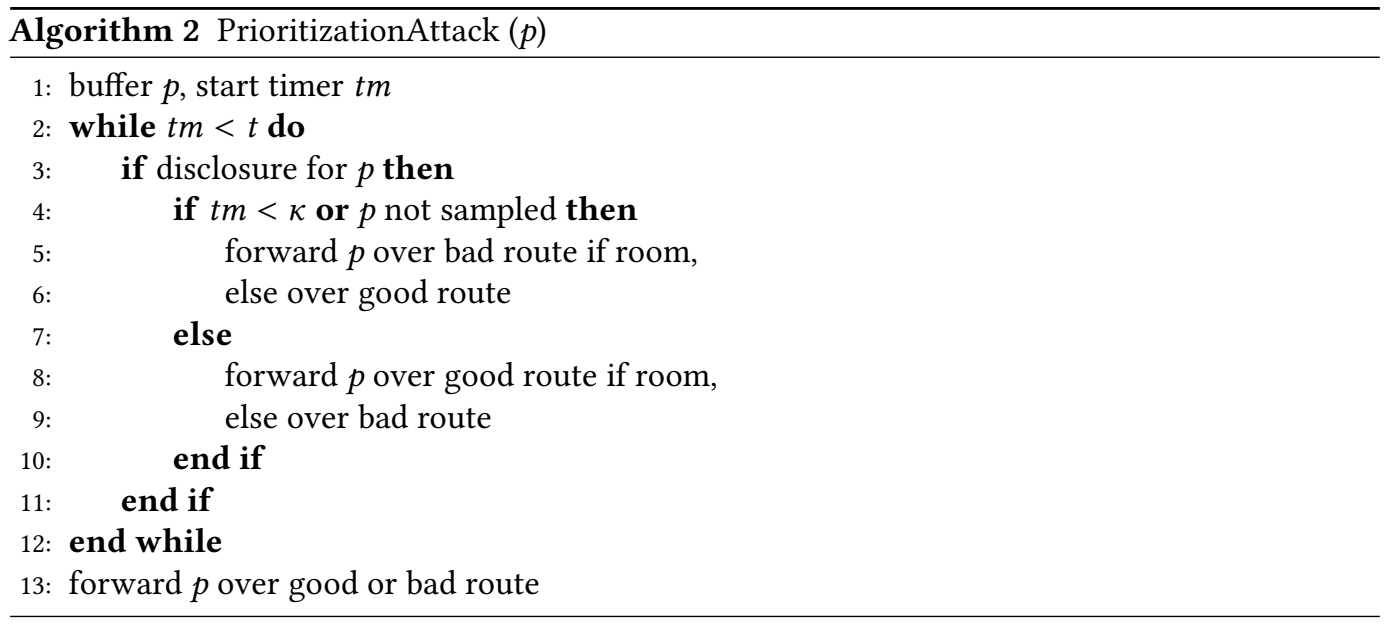

strategy (as long as, in the end, $i$ forwards a fraction $g$ of $F$ 's packets over the good route and the rest over the bad route).

By varying the parameters $t$ and $g$, we capture all rational behaviors of node $i$ that involve no more than two priority levels: (1) Honest behavior w/o prioritization: $t=0$ and $g=0$ ( $i$ does not buffer and forwards all packets on the same route). (2) Honest behavior w/ prioritization: $t=0$ and $g>0$ ( $i$ does not buffer and uses both routes). (3) Misbehavior: $t>0$ and $g>0$ ( $i$ buffers and uses both routes). For any behavior where node $i$ uses more than two priority levels (more than two routes), it is trivial to show that there exists a behavior where $i$ uses only two priority levels (e.g., only the best and worst of its routes) and domain $x$ achieves the same perceived performance. Hence, if we prove that any prioritization attack captured by the above model is unsuccessful, then we have also proved that any prioritization attack that uses more priority levels is unsuccessful.

The monitor estimates $x$ 's loss and delay with respect to $F$. When node $i$ is honest, the expected values of the mean loss and mean delay estimates are:

$$
\hat{l}_{\text {hon }}=g \cdot l_{g}+(1-g) \cdot l_{b}, \quad \hat{d}_{h o n}=g \cdot d_{g}+(1-g) \cdot d_{b},
$$

since a fraction $g$ of the traffic is forwarded over the good route and the rest over the bad route. When node $i$ misbehaves, we denote the expected values of the mean loss and delay estimates by $\hat{l}_{\text {mis }}$ and $\hat{d}_{\text {mis }}$, respectively.

We define the attack's "loss benefit" as $\hat{l}_{\text {hon }}-\hat{l}_{\text {mis }}$ and its "delay benefit" as $\hat{d}_{\text {hon }}-\hat{d}_{\text {mis }}$. These numbers quantify how much (on average) $x$ exaggerates its perceived loss and delay performance by misbehaving, taking into account that misbehavior involves buffering, which necessarily delays packets.

The attack's loss benefit is always positive: The longer node $i$ buffers packets before forwarding them, the bigger the fraction of packets whose sampling fate is disclosed to $i$ before forwarding. Ultimately, if $i$ does not mind introducing extra delay, there exist scenarios where it can buffer every single packet long enough to learn its sampling fate and forward all sampled packets over the good route and all non-sampled packets over the bad route. This would result in estimated mean loss rate $\hat{l}_{\text {mis }}=d_{g}$ and loss benefit $\hat{l}_{\text {hon }}-\hat{l}_{\text {mis }}=(1-g)\left(l_{b}-l_{g}\right)$.

The interesting question is what happens with the attack's delay benefit: under what conditions does the cost of buffering packets outweigh the benefit of forwarding packets based on their sampling fate? 


\subsection{Resistance to Prioritization}

We use terminology, notation, and tools from Palm calculus [5].

Let $F$ denote the stationary and ergodic process of flow $F$ 's packet arrivals at node $i$ (which has intensity $r$ ).

Let $P$ denote the stationary and ergodic process of all packet arrivals at node $i$ (which has intensity $R$ ).

Consider a packet $p$ from flow $F$ that arrives at node $i$ at an arbitrary point in time $\tau=0$, when $F$ and $P$ have run long enough to be in steady state.

Let $n_{F}[0, \kappa)$ denote the number of $F$-points that occur within time $\kappa$ after $p$ 's arrival.

Let $n_{F}\left[P_{0}, P_{\beta}\right)$ denote the number of $F$-points that occur within $\beta P$-points after $p$ 's arrival.

Palm calculus defines the "Palm probability of $p$ being sampled," which is the probability of $p$ being sampled as computed the moment of $p$ 's arrival.

LEMma 4.1. The Palm probability of p being sampled is:

$$
\mathbb{P}_{p}^{0}=\left(\left(1-\delta_{r}\right)^{n_{F}[0, \kappa)}-\left(1-\delta_{r}\right)^{n_{F}\left[P_{0}, P_{\beta}\right)}\right) \cdot \sigma
$$

Furthermore, if processes $F$ and $P$ satisfy the assumptions in $\S 2.5$, the distributions of $n_{F}[0, \kappa)$ and $n_{F}\left[P_{0}, P_{\beta}\right)$ do not depend on $p$ 's arrival time, and their expected values are:

$$
\mathbb{E}\left[n_{F}[0, \kappa)\right]=r \cdot \kappa, \quad \mathbb{E}\left[n_{F}\left[P_{0}, P_{\beta}\right)\right]=\frac{r}{R} \cdot \beta .
$$

\section{The proof is in the Appendix, §A.1.}

Lemma 4.1 says that node $i$ has no probabilistic benefit from treating a new packet $p$ that has just arrived preferentially, hence our algorithm is resistant to prioritization attacks that do not use buffering (use $t=0$ ). The intuition is that $p$ 's sampling fate depends only on future events (whether the if-statement on line 15 of Alg. 1 is true and whether disclosure for $p$ is early or late) which occur with the same probability for any just-arrived packet from the same flow. In particular, according to Equation 1, $p$ 's sampling fate depends on $\sigma, \delta_{r}, n_{F}[0, \kappa)$, and $n_{F}\left[P_{0}, P_{\beta}\right)$. The first two are fixed algorithm parameters. The last two are random distributions that govern, respectively, early and late disclosure, which do not depend on $p$ 's arrival time (because of the stationarity assumption [11]) and whose expectations (i.e., the best/mean-square-error predictions of their values) are the same for all new packets from flow $F$.

ClaIm 4.2. For any $t>0$ and $g \in(0,1)$, if:

$$
\begin{aligned}
\kappa & >d_{b}-d_{g}, \\
\left(1-\delta_{r}\right)^{r \kappa} & \geq \frac{1}{e},
\end{aligned}
$$

then:

$$
\hat{d}_{h o n}-\hat{d}_{m i s}<0
$$

Furthermore, if $t \leq \kappa$, then:

$$
\frac{d\left\{\hat{d}_{h o n}-\hat{d}_{m i s}\right\}}{d t} \leq \frac{d_{b}-d_{g}}{\kappa}-1 .
$$

The argument is in the Appendix, §A.2.

Claim 4.2 states sufficient conditions (Inequalities 2 and 3) under which: (1) The attack's delay benefit is always negative (Inequality 4), which means that the misbehaving domain $x$ always worsens its perceived delay performance. (2) The attack's delay benefit always decreases as the buffering period $t$ increases (Inequality 5), which means that the longer node $i$ buffers packets to 
gain information about their sampling fate, the worse $x$ 's perceived delay performance becomes. In particular, if $\kappa \gg d_{b}-d_{g}$, then $\frac{d\left\{\hat{d}_{\text {hon }}-\hat{d}_{\text {mis }}\right\}}{d t} \rightarrow-1$, which means that: for every msec that node $i$ buffers packets, it worsens $x$ 's perceived delay performance by almost one msec. In our opinion, given the importance of delay for modern applications, no domain would choose to penalize its perceived delay performance this way in order to exaggerate its loss performance.

Inequality 2 is straightforward: the quiet period should be longer than the delay difference between the bad and good route within the misbehaving domain. The longer the quiet period, the longer node $i$ needs to buffer $p$ before learning that $p$ will be sampled. By making the quiet period longer than the good-bad route delay difference, we ensure that $i$ cannot compensate for the buffering delay by sending $p$ over the good route.

Inequality 3 is more subtle and something we did not expect-it emerged from the math: the average fraction of packets that suffer early disclosure does not exceed $1-1 / e$. If disclosure for $p$ happens early (i.e., during a quiet period), node $i$ learns that $p$ will not be sampled and cheats by forwarding $p$ over the bad route. Increasing the quiet-period duration does not help control this event-quite the contrary: longer quiet periods lead to more early-disclosure events and more opportunities for this kind of cheating. Ultimately, the amount of packets that suffer early disclosure could become large enough to saturate the bad route; in this case, by forwarding all packets that suffer early disclosure over the bad route, node $i$ ends up forwarding all packets that do not suffer early disclosure-which include all the sampled packets-over the good route. Inequality 3 prevents this scenario, because it regulates/upper bounds (up to $1-1 / e$ ) the average fraction of packets that suffer early disclosure and could therefore be mistreated. This being a sufficient (not necessary) condition, we do not have an intuitive explanation for why the $1 / e$ bound in particular works; it is the tightest bound we could find that-together with Inequality 2-enabled us to prove that the delay benefit is always negative, but a tighter bound may exist.

\section{ACCURACY/TIMELINESS ANALYSIS}

When the monitor estimates the loss and delay experienced by an aggregate between two nodes, it can achieve any given target $(\gamma, \epsilon)$-accuracy as long as it waits long enough to collect the necessary sample size $N_{\gamma, \epsilon}$ (computed using standard statistics, as explained in the Appendix, §D.1). At the same time, we want to offer some notion of timeliness: for an aggregate of a given packet rate, collecting the necessary sample size should take a predictable, reasonable amount of time.

In this section, we establish a condition between $N_{\gamma, \epsilon}$, time interval $T$, and our algorithm parameters, such that a given node samples approximately $N_{\gamma, \epsilon}$ packets from a given flow $F$ within $T$.

Consider a flow $F$ that traverses a node $i$ and then another node $o$. F's packet arrivals at $i$ form a stationary, ergodic process that has intensity $r$. All packet arrivals at $i$ (resp. $o$ ) form a stationary, ergodic process that has intensity $R_{i}$ (resp. $R_{o}$ ). Node $i$ (resp. o) has buffer size $\beta_{i}$ (resp. $\beta_{o}$ ).

CLAIm 5.1. The expected number of packets that node $i$ samples from flow $F$, per time interval $T$, is approximately

$$
r \cdot T \cdot\left(\left(1-\delta_{r}\right)^{r \kappa}-\left(1-\delta_{r}\right)^{\frac{r}{R_{i}} \beta_{i}}\right) \cdot \sigma .
$$

\section{The argument is in the Appendix, §A.3.}

We obtain this term by applying Palm's inversion formula [5] to Equality 1, after approximating $n_{F}[0, \kappa)$ and $n_{F}\left[P_{0}, P_{\beta}\right)$ with their expected values. Given these approximations, the term is intuitive: $r \cdot T$ is the expected number of $F$ 's packets that arrive per $T ; \sigma$ is the probability that $i$ samples a packet $p$ from $F$ given that $p$ 's disclosure is neither early nor late; and the sum in parentheses 
approximates the probability that $p$ 's disclosure is neither early nor late. Approximating $n_{F}[0, \kappa)$ and $n_{F}\left[P_{0}, P_{\beta}\right)$ with their expected values is justified by our "fast ergodic convergence" assumption (§2.5). In the appendix, §A.3, we experimentally validate these approximations using real traffic traces.

Hence, if we want node $i$ to sample approximately $N_{\gamma, \epsilon}$ packets from flow $F$ within time interval $T$, it should be the case that

$$
N_{\gamma, \epsilon} \leq r \cdot T \cdot\left(\left(1-\delta_{r}\right)^{r \kappa}-\left(1-\delta_{r}\right)^{\frac{r}{R_{i}} \beta_{i}}\right) \cdot \sigma .
$$

Claim 5.2. If the following conditions hold:

I. nodes $i$ and o observe the same disclosure packets from flow $F$, and disclosure packets are not reordered with any other packets between $i$ and $o$;

II. the jitter between $i$ and $o$ is below $\mu$;

III. $\frac{\beta_{i}}{R_{i}}=\frac{\beta_{o}}{R_{o}}=\frac{\beta}{R}$;

then $i$ and o sample F's packets consistently.

\section{The argument is in the Appendix, §A.4.}

To be precise, the three conditions of Claim 5.2 ensure that the conditional probability that $o$ samples a packet $p$ given that $o$ observes $p$ and $i$ samples $p$ is approximately (not always) 1 . Condition I ensures that $i$ and $o$ pick the same disclosure packet for $p$. Condition II ensures that, if $p$ does not suffer early disclosure at $i$ and $i$ samples $p$, then $o$ will also sample $p$. Condition III ensures that, if $p$ does not suffer late disclosure at $i$, it will not suffer late disclosure at $o$ either.

If Conditions I and/or II do not hold ${ }^{1}$, the monitor can still achieve its target accuracy, but it needs to wait longer, because nodes produce consistent samples more slowly than indicated in Claim 5.2. We discuss this in the Appendix, §C.

\section{PARAMETRIZATION AND RESOURCES}

In this section, we describe how to parametrize our algorithm based on our misbehavior and accuracy/timeliness analyses $(\S 6.1)$ and state the resulting resource requirements $(\S 6.2)$.

\subsection{Algorithm Parametrization}

We parametrize our algorithm such that each node uses the minimum buffer size necessary and Inequalities 2, 3, and 6 are satisfied.

\section{Selection rate $\sigma$.}

Recall that $\sigma$ is the conditional probability with which a node samples a packet given that the packet does not suffer early or late disclosure at the node. Hence, $\sigma$ is the maximum sampling rate that our algorithm may apply to a flow. We set $\sigma$ according to the sampling capabilities of network devices. In the current Internet, we would set $\sigma$ to $1 \%$ or so, which is typically supported by modern network devices [6].

\section{Quiet period duration $\kappa$.}

We set $\kappa$ according to Inequality $2\left(\kappa \gg d_{b}-d_{g}\right)$, such that the delay benefit of any prioritization attack is negative and drops quickly as the buffering period increases. Given that we cannot know the value of $d_{b}-d_{g}$ for all prioritization attacks, we need to set $\kappa$ conservatively, such that it is significantly larger than any realistic intra-domain route difference. In the current Internet, we would set $\kappa$ to $100 \mathrm{msec}$ or so.

\footnotetext{
${ }^{1}$ Condition III is under our control, and we can make it hold.
} 


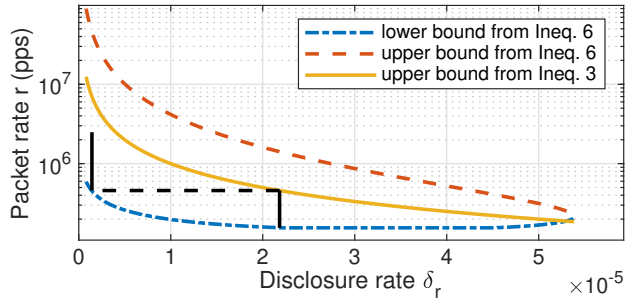

Fig. 3. Example operational regime.

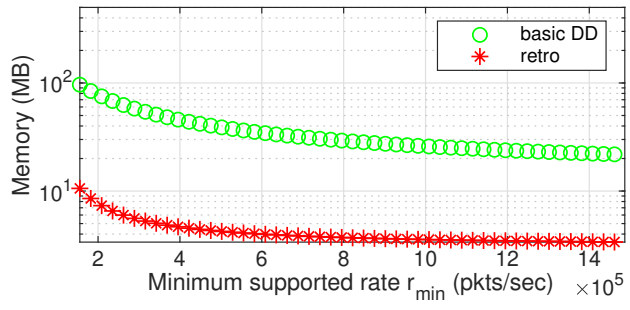

Fig. 4. Receipt-buffer size as a function of $r_{\text {min }}$.

\section{Buffer size $\beta$.}

(1) We pick the loss model (loss min $_{\text {m }}$ and-if we assume Gilbert loss-burst $t_{\max }$ ) and the target $(\gamma, \epsilon)$-accuracy that the monitor should achieve given this model. From these, we compute the minimum number of receipts $N_{\gamma, \epsilon}$ that the monitor needs to collect per flow in order to compute an estimate. The computation is in the Appendix, §D.1.

(2) We pick the time interval $T$, and the minimum and maximum flow intensity, $r_{\min }$ and $r_{\max }$, for which the monitor should achieve the target accuracy.

Given (1) and (2), we compute the minimum value of $\frac{\beta}{R}$ for which both Inequalities 3 and 6 are satisfied and set each node's buffer size accordingly. The computation is in the Appendix, §D.2.

There are two important things to note: First, for each node, we set the buffer size such that it is sufficient for all flow intensities in $\left[r_{\min }, r_{\max }\right]$. Second, across nodes, the buffer size can differ significantly, because it depends on the intensity $R$ of all packet arrivals at the node.

\section{Disclosure function $r \rightarrow \delta_{r}$.}

Recall that $\delta_{r}$ is the probability with which a node picks a packet from a flow of intensity $r$ as a disclosure packet.

For given values of $\sigma, \kappa$, and $\frac{\beta}{R}$, Inequalities 3 and 6 define the "operational regime" of our disclosure process: the set of all possible tuples $\left\{\delta_{r}, r\right\}$ that make prioritization attacks ineffective and make each node emit enough receipts in a timely manner. Fig. 3 shows an example operational regime: it is the surface enclosed between a lower bound obtained from Inequality 6 (blue curve) and the smallest of two upper bounds obtained, respectively, from Inequality 3 (yellow curve) and Inequality 6 (red curve); in this particular example, the smallest upper bound is the former (the yellow curve). The particular scenario for which we computed this operational regime does not matter for this discussion, but we provide it for completeness: target accuracy $(\gamma=95 \%, \epsilon=10 \%)$, target time interval $T=10 \mathrm{~min}$, minimum flow intensity $r_{\text {min }}=155 \mathrm{Kpps}$ (saturated OC-12 interface, assuming average packet size 500B), maximum flow intensity $r_{\max }=2.5 \mathrm{Mpps}$ (saturated OC-192 interface, assuming average packet size 500B), and algorithm parameters $\sigma=1 \%, \kappa=100 \mathrm{msec}$, and $\beta=10.5 \mathrm{MB}$.

We set our disclosure function such that any tuple $\left\{\delta_{r}, r\right\}$ falls in the operational regime; we describe how in the Appendix, §D.3. Fig. 3 shows an example disclosure function (black curve) that consists of two vertical lines: all flow intensities below $437 \mathrm{Kpps}$ map to disclosure rate $2.18 \cdot 10^{-5}$, while all flow intensities above $437 \mathrm{Kpps}$ map to disclosure rate $0.137 \cdot 10^{-5}$.

\subsection{Resource Requirements}

Having established how to parametrize our algorithm, we considered the values we would use in various realistic scenarios and computed the resulting resource requirements. 


\section{Memory overhead.}

We found that our algorithm requires a buffer size that increases data-path memory only by a few percentage points, which is, in most cases, about an order of magnitude less than basic delayed disclosure (DD).

We present a concrete example: Consider a node collocated with a $10 \mathrm{GigE}$ interface, observing traffic of intensity $R=2.5 \mathrm{Mpps}$ with an average packet size 500B. Assume 12B per receipt (§E.1). We set $\sigma=1 \%, \kappa=100 \mathrm{msec}$, loss $_{\min }=0.1 \%, \gamma=95 \%, \epsilon=10 \%$, and $T=10 \mathrm{~min}$.

Fig. 4 shows the buffer size required by our algorithm versus basic DD as a function of $r_{\min }$ (the minimum supported flow intensity). Our algorithm (red stars, abbrv. "retro") requires a few MB of data-path memory, whereas basic DD (green circles) requires about an order of magnitude more. To put this in perspective, a 10GigE interface needs about $125 \mathrm{MB}$ of packet buffers (using the "one round-trip worth of traffic" rule and assuming a typical Internet round-trip of $100 \mathrm{msec}$ ), i.e., our algorithm increases data-path memory by only a few percentage points.

There are three reasons why our algorithm requires less data-path memory than basic DD: (1) Basic DD was designed to collect a target fraction of packets from each flow, not a target sample size in a target time interval; as a result, it samples more packets than necessary. (2) Basic DD uses a fixed disclosure rate $\delta$, yet no single $\delta$ works well for all flows: faster flows need a lower $\delta$ to prevent early disclosure from happening too often, while slower flows need a higher $\delta$ to prevent late disclosure from happening too often. Basic DD picks a $\delta$ that is low enough to accommodate the fastest flows (those with intensity close to $r_{\max }$ ) and, as a result, requires too much memory to protect the slowest flows (those with intensity close to $r_{\text {min }}$ ) from late disclosure. (3) Basic DD avoids late disclosure with a fixed, high probability; as a result, it avoids late disclosure more than necessary to achieve the target accuracy in the target time interval.

\section{Processing overhead.}

Our algorithm requires a small number of hash computations, timestamp comparisons, and accesses to data-path memory per packet, which is similar to basic DD (we claim no improvement on processing overhead). The exact numbers depend on the implementation. The hardware design sketched in the Appendix, §E.2, requires per packet: two hash computations, a couple of timestamp comparisons, one read and one write access to data-path SRAM, and a parallel lookup and update to data-path CAM.

\section{EXPERIMENTAL EVALUATION}

After describing our methodology (§7.1), we demonstrate that our algorithm is useful (§7.2) and confirm that it works as expected (§7.3) and is resistant to prioritization attacks (§7.4).

\subsection{Methodology}

In each experiment, we emulate some number of target flows, crossing one or more domains. Each target flow enters each domain at one node and exits the domain at another node; there is no packet reordering between the nodes.

We use 1-hour backbone traces made available by CAIDA in 2016 (chicago-equinix, direction A). Each flow observed at an entry node consists of one entire trace, while the total traffic observed at an entry node consists of multiple traces merged into one. Why this particular emulation: The question we are most frequently asked is how well our system would work for busy backbone routers located at the Internet core. The traffic rate of a single CAIDA trace ranges from a few hundred Mbps to a few Gbps; this is too low to represent the total traffic arriving at a busy backbone-router interface, but could represent, e.g., the traffic between a source and destination prefix connected 
to the Internet through OC-48 or lightly loaded 10GigE links. By merging multiple traces-and shifting packet timestamps such that all traces start at the same time-we created higher-rate ingress streams.

In each experiment, we emulate either i.i.d. or bursty loss (of various rates). For the latter, we obtained the loss pattern from an actual congested link: we created in our lab a simple topology where 16 pairs of end-points communicated over a bottleneck GigE link, and we had each pair exchange back-to-back TCP flows; this resulted in the bottleneck link experiencing packet loss of average rate $4.8 \%$ and burstiness 1.52 packets.

Our configuration is purposefully not realistic in all scenarios, because we want our algorithm to operate at its limits. For instance, in a real deployment, we would set $l o s s_{\min }=0.1 \%$ or so. However, if the actual loss rate is $\gg$ loss $_{\text {min }}$, our algorithm will use significantly more memory than necessary to estimate this loss rate, and our results will be obviously good. Hence, in each experiment, we set loss $_{\min }$ to the actual loss rate introduced in the experiment, which results in our algorithm using the minimum memory needed to estimate this loss rate. So:

(1) In all experiments, we set the selection rate to $\sigma=1 \%$, the quiet-period duration to $\kappa=$ $100 \mathrm{msec}$, the target accuracy to $(\gamma=95 \%, \epsilon=10 \%)$, and the target time interval to $T=5 \mathrm{~min}$.

(2) In all experiments, we set loss $_{\min }$ to the actual loss rate and-when the actual loss is burstyburst $_{\text {max }}$ to the actual loss burstiness. This way, we test how accurately the monitor estimates loss that is at the limit of what the nodes were configured to handle.

(3) In $§ 7.2$ and $§ 7.3$, we set the minimum supported flow intensity $r_{\min }$ to the average packet rate of the slowest flow involved in the experiment. This way, we test how accurately the monitor estimates loss experienced by a flow whose packet rate is at the limit of what the nodes were configured to handle.

(4) In §7.4, where node $i$ launches prioritization attacks, we configure the nodes such that the average packet rate of the target flow $F$ falls on the upper bound of the operational regime (the middle, yellow curve in Fig 3). This is the most challenging setting we could think of: if node $i$ operates close to the upper bound of the operational regime, it is possible that $F$ 's instant packet rate fluctuates so fast that it temporarily pushes node $i$ out of the operational regime, where it could potentially cheat.

\subsection{Use Cases}

First, we demonstrate that our algorithm enables the monitor to draw useful conclusions about network behavior.

In each experiment, we emulate two to four flows that enter an ISP $x$ at node $i$ and exit at node $o$. Both nodes are collocated with highly loaded $10 \mathrm{GigE}$ interfaces. We use four CAIDA traces. The total traffic observed at node $i$ consists of the four traces merged together and has a rate of $8 \mathrm{Gbps}$.

\section{SLA verification.}

ISP $x$ has signed an SLA with a customer network, promising loss below $0.1 \%$; the customer's traffic suffers exactly $0.1 \%$ loss within $x$. On the customer's request, the monitor estimates $x$ 's loss with respect to traffic from the customer's prefix to four popular destination prefixes (so, we have four aggregates, each corresponding to a flow). Fig. 5a shows the monitor's loss estimates with respect to each aggregate/flow after the target $5 \mathrm{~min}$ time interval; in each boxplot, the red line shows the median, while the limits of the boxplot indicate the $95 \%$ confidence interval. We see that all estimates achieve the target accuracy $(\gamma=95 \%, \epsilon=10 \%)$. Hence, $y$ correctly determines that $x$ is borderline violating its SLA. 


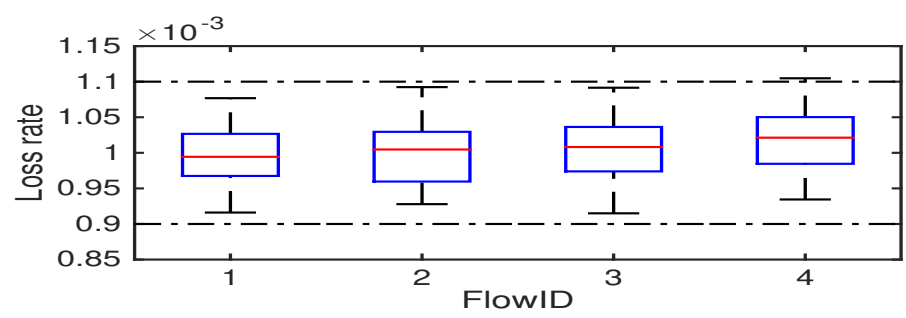

(a) I.i.d. loss $0.1 \%$.

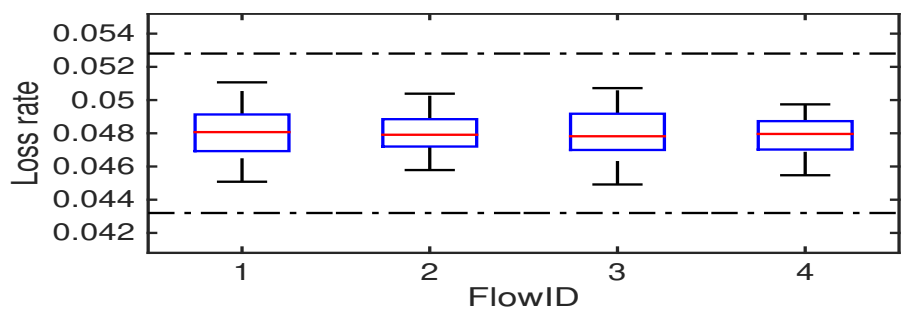

(b) Bursty loss $4.8 \%$.

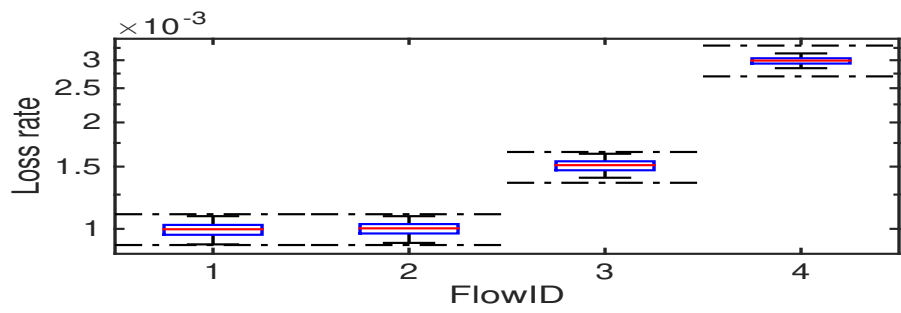

(c) Loss rates per flow ID: $0.1 \%, 0.1 \%, 0.15 \%$ and $0.3 \%$.

Fig. 5. Loss estimates after $T=5 \mathrm{~min}$.

\section{Cheap SLA verification.}

Same as above, but this is a cheaper SLA, promising loss below 5\%; the customer's traffic suffers $4.8 \%$ loss with burstiness 1.5 due to a congested bottleneck link at $x$ 's core. Fig. $5 \mathrm{~b}$ shows that the monitor's loss estimates achieve the target accuracy after the target $5 \mathrm{~min}$ time interval. Hence, $y$ correctly determines that $x$ is honoring its SLA, despite the fact that it is introducing loss just below the promised maximum.

\section{Subtle prefix discrimination.}

Transit ISP $x$ is dissatisfied with networks $y$ and $z$ : the former is a popular video provider; the latter is an eyeball ISP whose customers freely participate in peer-to-peer networks; $x$ wants to renegotiate its peering agreement with each of these networks, but they are resisting. In response, $x$ subtly discriminates against them: while most transit traffic experiences loss $0.1 \%, y$ 's and $z$ 's traffic experience, respectively, $0.15 \%$ and $0.3 \%$ loss. After user complaints, the monitor estimates $x$ 's loss with respect to two random flows, one flow originating from $y$, and one flow originating from $z$ (so, again, we have four aggregates, each corresponding to a flow). Fig. $5 \mathrm{c}$ shows that the monitor catches the discrimination after the target $5 \mathrm{~min}$ time interval.

\section{Policing of SYN packets.}

ISP $x$ has signed an SLA with a customer network, promising loss below 0.1\%; it honors this SLA for all but TCP SYN packets, which are policed such that they experience 3-30 times higher loss (we conducted 240 experiments with various policing rates). The customer observes end-to-end 


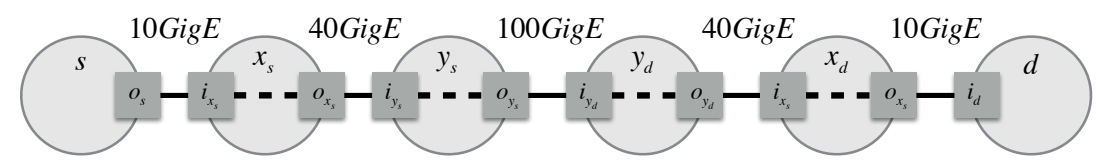

Fig. 6. Topology emulated in $§ 7.3$.

that something is wrong with connection setup and asks the monitor whether $x$ is discriminating against its SYN packets. In response, the monitor defines two aggregates: SYN packets from the customer's prefix to some popular destination prefix; all other packets with the same source and destination prefix. So, in this case, we have two aggregates that are subsets of the same flow.

The challenge is that the SYN aggregate is relatively small, and the monitor would need to collect receipts for hours in order to estimate $x$ 's loss with respect to the SYN aggregate with the target accuracy of $(y=95 \%, \epsilon=10 \%)$. However, the goal here is not to estimate $x$ 's performance, but to determine whether it treated the two aggregates differently. This can be done much faster, with a simple Maximum Likelihood differentiation detector: the monitor estimates $x$ 's loss rate for each aggregate based on the receipts it collects within some period of time (minutes, not hours); and computes the corresponding confidence intervals (CI); if the lower limit of the CI for the SYN aggregate estimate is greater than the upper limit of the CI for the no-SYN aggregate, then the monitor concludes that $x$ discriminates against the SYN aggregate.

Our results, after the target $5 \mathrm{~min}$ time interval: When $x$ 's loss with respect to SYN packets is 5 or more times higher, the monitor detects differentiation with probability $100 \%$ (in all experiment runs); when SYN loss is 3-5 times higher, detection rate is $\geq 94 \%$; for subtler differentiation, detection rate drops sharply. For completeness, we also ran 480 experiments where $x$ does not differentiate, and the monitor correctly detects no differentiation.

\subsection{Basic Operation}

Next, we confirm that our algorithm works as it should, i.e., the nodes sample consistently at the rate they are supposed to.

We emulate the topology in Fig. 6: there are 4 flows between edge networks $s$ and $d ; 6$ additional flows enter and exit the topology, respectively, at regional ISPs $x_{s}$ and $x_{d}$; and 22 additional flows enter and exit, respectively, at Tier-1 ISPs $y_{s}$ and $y_{d}$. Hence, we use a total of 32 CAIDA traces. On average, nodes $o_{s}, o_{x_{s}}$, and $o_{y_{s}}$ observe, respectively, 1.92, 4.81 and 15.47Mpps (equiv. 7.67, 19.2 and $61.8 \mathrm{Gbps}$ ). There is i.i.d. loss of rate $0.2 \%$ inside domain $x_{s}$ and $0.1 \%$ inside domain $y_{s}$.

Fig. 7 shows, for each flow, the number of packets that are consistently sampled by all the nodes that observe the flow within the target time interval. For instance, Fig. $7 \mathrm{a}$ shows the number of consistently sampled packets for each of the 4 flows observed by all 10 nodes; Fig. 7c shows the number of consistently sampled packets for each of the 32 flows observed by nodes $o_{y_{s}}$ and $i_{y_{d}}$. In all plots, the horizontal red line shows the minimum sample size $N_{\gamma, \epsilon}$ needed to estimate i.i.d. loss of minimum rate loss $_{\min }=0.1 \%$ with the target accuracy.

We see that, for all flows, the produced consistent sample size exceeds the minimum necessary. In particular, Fig. 7a shows that all 10 nodes sample consistently at the necessary rate, despite the fact that they observe packet-arrival intensities that differ by an order of magnitude; this is because each node's receipt buffer is large enough to accommodate the packet-arrival intensity that the node may observe. Moreover, Fig. 7c shows that nodes $o_{y_{s}}$ and $i_{y_{d}}$ sample consistently at the necessary rate from all 32 flows that they observe in common; this is because each node is configured to produce at least the minimum sample size for all flows with intensity $\geq r_{\text {min }}$. 


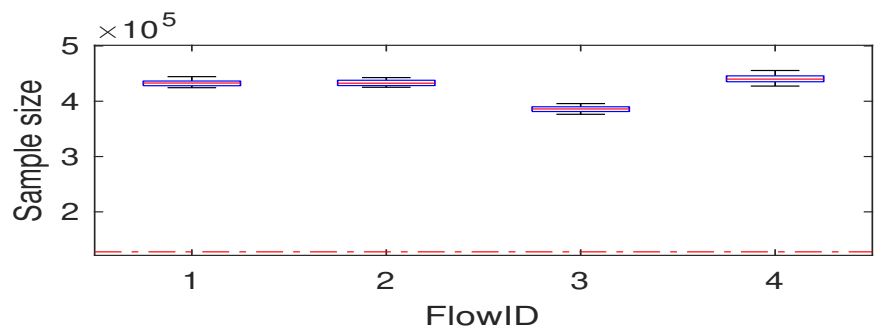

(a) Flows between $s$ and $d$.

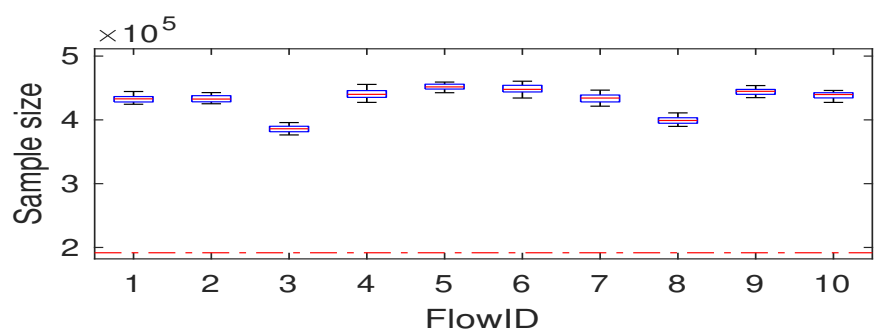

(b) Flows between $x_{s}$ and $x_{d}$.

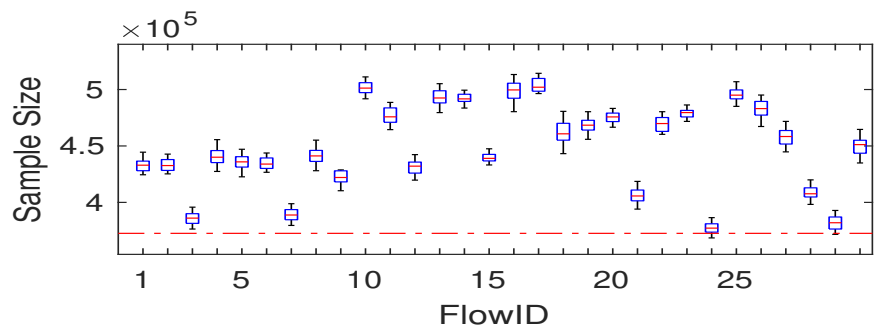

(c) Flows between $y_{s}$ and $y_{d}$.

Fig. 7. Consistent sample size after $T=5 \mathrm{~min}$.

\subsection{Resistance to Prioritization}

Finally, we confirm that our algorithm makes prioritization attacks ineffective, whereas basic delayed disclosure (DD) allows a misbehaving network to significantly exaggerate its performance.

ISP $x$ launches a prioritization attack (\$4.1) against one target flow. The delay of the two routes is $d_{b}=50 \mathrm{msec}$ and $d_{g}=10 \mathrm{msec}$ (the good route is 5 times faster than the bad one). We vary the attack parameter $g$ (the fraction of traffic that fits in the good route) from 0 to $100 \%$, and the attack parameter $t$ (the buffering period) from 0 to $120 \mathrm{msec}$. We measure the attack's relative delay benefit: by how much the misbehaving domain exaggerates its perceived delay performance relative to the delay performance it would achieve without a prioritization attack.

Fig. 8a shows the results. The $y$-axis shows the attack's relative delay benefit (in percentage points), while the two $x$-axes show, respectively, the attack parameters $t$ (in msec) and $g$ (in percentage points). We do not obtain the plotted data points from our formulas but experimentally, as the monitor would compute them from the receipts produced by x's entry and exit nodes.

We see that the attack's relative delay benefit is always negative and as low as $-1122 \%$ (by misbehaving, $x$ worsens its perceived delay performance by this much). The highest delay benefit is close to 0 , and it occurs when $t \rightarrow 0$, at which point $x$ almost does not buffer/cheat. The lowest delay benefit occurs at $t=120 \mathrm{msec}$ and $g \rightarrow 1$. At this point, $x$ forwards most packets over the $10 \mathrm{msec}$ good route, while it buffers sampled packets for $120 \mathrm{msec}$. As a result, relative to an honest 


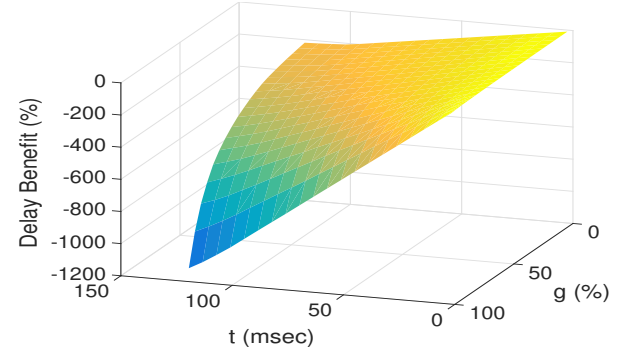

(a) Our algorithm.

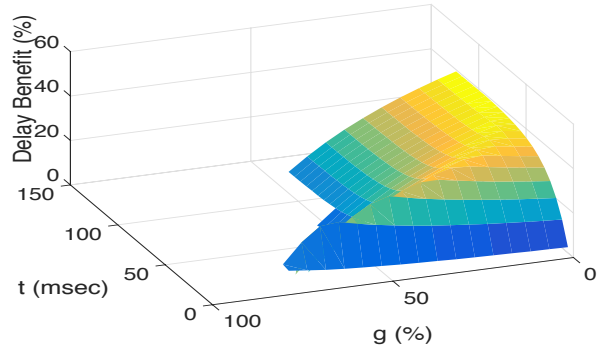

(b) Basic delayed disclosure.

Fig. 8. Relative delay benefit of a prioritization attack as a function of attack parameters $t$ and $g$.

behavior (where it would not buffer and its performance, both true and perceived, would be almost $10 \mathrm{msec}$ ), $x$ worsens its relative delay benefit by almost $1200 \%$. This is consistent with the math in the proof of Lemma 4.2, which shows that $x$ 's misbehaving function is convex w.r.t. $t$ and concave w.r.t. $g$, hence the lowest delay benefit occurs when either $g \rightarrow 0$ or $g \rightarrow 1$.

One might expect that basic DD would also handle prioritization attacks well, without the added complexity of quiet periods and an adaptive disclosure rate. To show that this is not the case, we repeat the experiment, but have node $i$ run basic DD instead of our algorithm. To cheat, node $i$ buffers each packet $p$ for $t$ or until disclosure occurs, whichever comes first; if disclosure comes first and $p$ is sampled, node $i$ forwards $p$ over the good route, otherwise, it forwards $p$ such that, overall, a fraction $g$ of all packets are forwarded over the good route. We configure basic DD with $\beta=4.2 \mathrm{MB}$ (the same amount used by our algorithm) and $\delta=9.9 * 10^{-5}, \sigma=1 \%$ (as advised in [3]).

We see, in Fig. 8b, that there exist multiple prioritization strategies-multiple $\{g, t\}$ combinationswhere the attack's delay benefit is both positive and significant, as high as $+41 \%$ (by misbehaving, $x$ improves its perceived delayed performance by this much). The intuition is that $x$ does not need to know the sampling fate of all the packets it forwards in order to exaggerate its perceived delay performance; knowing the sampling fate of a small fraction of the forwarded packets is enough, and this knowledge can be gained with a surprisingly short $t$ that does not introduce significant delay overhead. As a result, without our counter-measures, prioritization attacks defeat the purpose of delayed disclosure.

\section{RELATED WORK}

The closest work is Network Confessional [3], which shares the same goals; we take from it the idea of delayed disclosure, but show that that idea alone is vulnerable to prioritization attacks and requires expensive, suboptimal resources on the data-path.

Apart from Network Confessional, delayed disclosure has been mentioned twice in the context of fault localization $[24,25]$. In both proposals, there is no in-band disclosure process: sampling nodes are explicitly told which packets to sample, either by end-hosts [24] or by a central controller [25]. Neither approach would work for an Internet-wide transparency system-we cannot imagine a secure, scalable system where backbone routers handle end-host or controller disclosure messages for individual packets. Neither work explores the idea in depth or provides misbehavior, accuracy, or resource analysis.

Beyond delayed disclosure, there exists extensive related work on fault localization $[1,2,4,14,26]$. In the first proposals, nodes produce per-packet [1] or per-TCP-flow [2] receipts, which may be fine for low-rate environments but violate our low-resource-cost goal. Subsequent work brought formal rigor and security guarantees to the idea of networks reporting on their own performance $[4,14,26]$. 
These proposals show that any fault can be localized to a link between two subsequent reporting nodes-even when there is no centralized monitor, and networks may tamper both with packet contents and with the receipts produced by other networks. However, these proposals focus on fault localization for specific "paths" (in our context, a path would correspond to a flow) and require nodes to keep per-path state. This is something that we cannot afford given our goals $(\S 2.2, \S 2.3)$.

\section{CONCLUSIONS}

We proposed a packet sampling algorithm that prevents the network node that performs the sampling from treating the sampled packets preferentially. Our algorithm is a building block for a network transparency system, where domains produce receipts for a small sample of observed packets, and an independent monitor collects each domain's receipts and uses them to estimate the domain's mean loss rate and delay distribution quantiles. Our algorithm builds on delayed disclosure-where the sampling function is disclosed to the sampling node with a delay-and enhances it with quiet periods, during which sampling is disallowed, and a disclosure process that adapts to each flow's packet rate. These two techniques together ensure that a misbehaving domain that tries to bias the sample to exaggerate its perceived performance instead worsens it. Our algorithm can be configured to emit enough receipts to achieve a target accuracy within a target time interval, while using the minimum amount of data-path memory necessary-which ends up being a few MB of data-path memory per 10Gbps of forwarding capacity.

Acknowledgments. We deeply thank Ovidiu Mara, who did experiments to help us calibrate our bursty loss model, the SIGMETRICS reviewers for their feedback, and our shepherd, Daniel Figueiredo, for his extraordinary patience and level of involvement that helped significantly improve our paper. 


\section{REFERENCES}

[1] Katerina Argyraki, Petros Maniatis, David Cheriton, and Scott Shenker. 2004. Providing Packet Obituaries. In Proc. of the ACM Workshop on Hot Topics in Networking (HotNets).

[2] Katerina Argyraki, Petros Maniatis, Olga Irzak, Subramanian Ashish, and Scott Shenker. 2007. Loss and Delay Accountability for the Internet. In Proc. of the IEEE International Conference on Network Protocols (ICNP).

[3] Katerina Argyraki, Petros Maniatis, and Ankit Singla. 2010. Verifiable Network-performance Measurements. In Proc. of the International Conference on emerging Networking EXperiments and Technologies (CoNEXT).

[4] Boaz Barak, Sharon Goldberg, and David Xiao. 2008. Protocols and Lower Bounds for Failure Localization in the Internet. In Proc. of the International Conference on the Theory and Applications of Cryptographic Techniques (EUROCRYPT).

[5] Jean-Yves Le Boudec. 2011. Performance Evaluation of Computer and Communication Systems. EFPL Press.

[6] Cisco. 2019. IOS NetFlow. Retrieved January 2019 from http://www.cisco.com/c/en/us/products/ios-nx-os-software/ ios-netflow/index.html

[7] Global Net Neutrality Coalition. 2019. Status of Net Neutrality Around the World. Retrieved January 2019 from https://www.thisisnetneutrality.org/

[8] Cogent. 2016. Network Services SLA Global. Retrieved January 2019 from https://cogentco.com/files/docs/network/ performance/global_sla.pdf

[9] Comcast. 2009. Service Level Agreement for Wholesale Dedicated Internet. Retrieved January 2019 from https://portals.comcasttechnologysolutions.com/sites/default/files/service_level_agreement_for_wholesale_ dedicated_internet_sla07292014.pdf

[10] European Commission. 2013. On-line public consultation on "specific aspects of transparency, traffic management and switching in an Open Internet”. Retrieved January 2019 from https://ec.europa.eu/digital-single-market/en/news/ answers-public-consultation-specific-aspects-transparency-traffic-management-and-switching-open

[11] David Cox and P A. W. Lewis. 1966. The statistical analysis of series of events. Springer. 59-60 pages.

[12] DPDK. 2015. Data Plane Development Kit. Retrieved May 2015 from http://dpdk.org

[13] Nick Duffield and Matthias Grossglauser. 2001. Trajectory Sampling for Direct Traffic Observation. IEEE/ACM Transactions on Networking (June 2001).

[14] Sharon Goldberg, David Xiao, Eran Tromer, Boaz Barak, and Jennifer Rexford. 2008. Path-quality Monitoring in the Presence of Adversaries. In Proc. of the ACM International Conference on Measurement and Modeling of Computer Systems (SIGMETRICS).

[15] Shay Gueron. 2010. Intel Advanced Encryption Standard (AES) New Instruction Set. Retrieved January 2019 from https://software.intel.com/sites/default/files/article/165683/aes-wp-2012-09-22-v01.pdf

[16] Gerhard Hasslinger and Oliver Hohlfeld. 2008. The Gilbert-Elliott Model for Packet Loss in Real Time Services on the Internet. In Proc. of the GI/ITG Conference - Measurement, Modelling and Evalutation of Computer and Communication Systems $(M M B)$.

[17] Manish Joshi and Theyazn Hassn Hadi. 2015. A Review of Network Traffic Analysis and Prediction Techniques. (2015). arXiv:arXiv:1507.05722

[18] Myungjin Lee, Nick Duffield, and Ramana Rao Kompella. 2010. Two Samples Are Enough: Opportunistic Flowlevel Latency Estimation Using Netflow. In Proc. of the IEEE International Conference on Computer Communications (INFOCOM).

[19] William Lehr, Erin Kenneally, and Steven Bauer. 2015. The Road to an Open Internet is Paved with Pragmatic Disclosure and Transparency Policies. In Proc. of the Telecommunications Policy Research Conference (TPRC).

[20] Body of European Regulators for Electronic Communications (BEREC). 2016. BoR (16) 127: Guidelines on the Implementation by National Regulators of European Net Neutrality Rules. Retrieved January 2019 from http://berec.europa.eu/ eng/document_register/subject_matter/berec/download/0/6160-berec-guidelines-on-the-implementation-b_0.pdf

[21] Christos Pappas, Katerina Argyraki, Stefan Bechtold, and Adrian Perrig. 2015. Transparency Instead of Neutrality. In Proc. of the ACM Workshop on Hot Topics in Networks (HotNets).

[22] Joel Sommers, Paul Barford, Nick Duffield, and Amos Ron. 2007. Accurate and Efficient SLA Compliance Monitoring. SIGCOMM Computer Communication Review (Oct 2007).

[23] Verizon. 2019. Global Latency and Packet Delivery SLA. Retrieved January 2019 from http://www.verizonenterprise. com/terms/global_latency_sla.xml

[24] Xin Zhang, Abhishek Jain, and Adrian Perrig. 2008. Packet-dropping Adversary Identification for Data Plane Security. In Proc. of the International Conference on emerging Networking EXperiments and Technologies (CoNEXT).

[25] Xin Zhang, Chang Lan, and Adrian Perrig. 2012. Secure and Scalable Fault Localization Under Dynamic Traffic Patterns. In Proc. of the IEEE Symposium on Security and Privacy (SP).

[26] Xin Zhang, Zongwei Zhou, Hsu-Chun Hsiao, Adrian Perrig, and Patrick Tague. 2012. ShortMAC: Efficient Data Plane Fault Localization. In Proc. of the NDSS Symposium (NDSS). 
[27] Tanja Zseby and Sebastian Zander. 2001. Evaluation of Building Blocks for Passive One-way-delay Measurements. In Proc. of the workshop on Passive and Active Measurement (PAM).

\section{A PROOFS}

\section{A.1 Proof of Lemma 4.1}

Palm sampling probability. Let $X_{p}$ denote the number of $F$-points until $p$ 's disclosure packet arrives (which is also an $F$-point occurrence). $n_{F}[0, \kappa)$ denotes the number of $F$-points that occur within a time interval $\kappa$ after $\tau=0 ; n_{F}\left[P_{0}, P_{\beta}\right)$ denotes the number of $F$-points that occur within the upcoming $\beta P$-points after $p$.

Packet $p$ is sampled when the following three events occur: (a) the disclosure of $p$ is not early, i.e., $X_{p}>n_{F}[0, \kappa)$; (b) neither is it late, i.e., $X_{p} \leq n_{F}\left[P_{0}, P_{\beta}\right)$; and (c), $p$ is selected by line 15 of Alg. 1 . Therefore, $p$ 's sampling probability $\mathbb{P}^{0}(a, b, c)$, as computed at time $\tau=0$ is given by the chain rule of probability:

$$
\mathbb{P}^{0}(a, b, c)=\mathbb{P}(a) \cdot \mathbb{P}(b \mid a) \cdot \mathbb{P}(c \mid a, b)
$$

The last part $\mathbb{P}(c \mid a, b)$ of Eq. 7 is equal to the selection rate $\sigma$. The first two parts are computed based on the following observation: In Alg. 1, DiscHash has strong randomization properties, which means that the arrivals of the disclosure packets occur independently over the $F$-arrivals and with equal probability $\delta_{r}$. I.e., the disclosure arrivals form a Bernoulli stochastic process on the top of $F$-arrivals, which is renewed at each $F$-point. Therefore, for any arbitrary packet $p$ of flow $F$, the "distance" until the disclosure packet $X_{p}$, counted in $F$-points, follows the geometric distribution:

$$
\begin{aligned}
& \mathbb{P}(a) \cdot \mathbb{P}(b \mid a)= \\
& =\mathbb{P}\left(X_{p}>n_{F}[0, \kappa)\right) \cdot \mathbb{P}\left(X_{p} \leq n_{F}\left[P_{0}, P_{\beta}\right) \mid X_{p}>n_{F}[0, \kappa)\right) \\
& =\mathbb{P}\left(X_{p}>n_{F}[0, \kappa)\right) \cdot\left(1-\mathbb{P}\left(X_{p}>n_{F}\left[P_{0}, P_{\beta}\right) \mid X_{p}>n_{F}[0, \kappa)\right)\right) \\
& =\mathbb{P}\left(X_{p}>n_{F}[0, \kappa)\right) \cdot\left(1-\mathbb{P}\left(X_{p}>n_{F}\left[P_{0}, P_{\beta}\right)-n_{F}[0, \kappa)\right)\right) \\
& =\left(1-\delta_{r}\right)^{n_{F}[0, \kappa)} \cdot\left(1-\left(1-\delta_{r}\right)^{n_{F}\left[P_{0}, P_{\beta}\right)-n_{F}[0, \kappa)}\right) \\
& =\left(1-\delta_{r}\right)^{n_{F}[0, \kappa)}-\left(1-\delta_{r}\right)^{n_{F}\left[P_{0}, P_{\beta}\right)},
\end{aligned}
$$

where the third derivation step is because of the memoryless property of the geometric distribution.

Given Eq. 7 and 8, the sampling probability of packet $p$ is:

$$
\mathbb{P}^{0}(a, b, c)=\left(\left(1-\delta_{r}\right)^{n_{F}[0, \kappa)}-\left(1-\delta_{r}\right)^{n_{F}\left[P_{0}, P_{\beta}\right)}\right) \cdot \sigma
$$

Independence from p's arrival time. This is an immediate consequence of the stationarity assumption made in Section §2.5. Stationarity of point processes implies that: the distribution of the number of points in a fixed interval $\left(\tau_{1}^{\prime}, \tau_{1}^{\prime \prime}\right]$ is invariant under translation, i.e., is the same for $\left(\tau_{1}^{\prime}+h, \tau_{1}^{\prime \prime}+h\right]$ for all $h$. It depends only on the length of the interval and not its time origin [11]. Therefore, the distributions of the number of points $n_{F}[0, \kappa)$ and $n_{F}\left[P_{0}, P_{\beta}\right)$ depend only on $\kappa$ and $\beta$ and not on the time that $p$ is observed. They are the same at any arbitrary point in time $\tau=0$.

Expected values of $n_{F}\left[P_{0}, P_{\beta}\right)$ and $n_{F}[0, \kappa)$. The expected values of $n_{F}[0, \kappa)$ and $n_{F}\left[P_{0}, P_{\beta}\right)$ are computed using Palm Calculus [5]:

$$
\begin{aligned}
\mathbb{E}\left[n_{F}[0, \kappa)\right] & =r \cdot \kappa \\
\mathbb{E}\left[n_{F}\left[P_{0}, P_{\beta}\right)\right] & =\frac{r}{R} \cdot \beta
\end{aligned}
$$


Eq. 10 is an immediate consequence of the definition of the intensity of a stationary point process, which is equal to the expected number of points per time unit. Eq. 11 requires the following auxiliary lemma A.1, and linearity of expectation.

Lemma A.1. Given two point processes $P$ and $F$ of the same stationary process, which have rates $R$ and $r$, respectively, the probability of an arbitrary $P$-arrival to be an $F$-arrival (i.e the arrived packet belongs to flow $F$ ), is:

$$
\pi_{F}=\frac{r}{R}
$$

Proof: $P$ and $F$ are two point processes of the same stationary continuous-time arrival process. Their intensities $(R$ and $r$ ) are just two different event "clocks" of that process: $P$-clock ticks every time that a packet arrives and $F$-clock ticks only if the arriving packet belongs to flow $F$. Let $\lambda_{F}(P)$ denote the intensity of the $P$ point process measured with the event clock $F$. Also, let $n_{F}\left[P_{0}, P_{1}\right]$ denote the number of points of process $F$ that fall between the two random subsequent $P$ points $P_{0}$ and $P_{1}$.

We apply Neveu's Exchange Theorem [5] to obtain:

$$
\begin{aligned}
\lambda_{F}(P) & =\frac{R}{r} \\
\mathbb{E}\left[n_{F}\left[P_{0}, P_{1}\right)\right] & =\frac{1}{\lambda_{F}(P)} .
\end{aligned}
$$

Note that $n_{F}\left[P_{0}, P_{1}\right)$ is 1 , if the packet at $P_{0}$ is an $F$ packet (i.e., if the $P$-point at $P_{0}$ is also an $F$-point), and 0 otherwise. Hence, $\mathbb{E}\left[n_{F}\left[P_{0}, P_{1}\right)\right]$ is the probability $\pi_{F}$ that an arbitrary packet is a packet from flow $F$. Thus:

$$
\pi_{F}=\frac{\lambda(F)}{\lambda(P)}=\frac{r}{R}
$$

Three notes are important:

Note 1: The geometric distribution requires only an integer exponent of $\left(1-\delta_{r}\right)$. This is because in the PMF of a geometrically distributed random variable the exponent denotes the number of trials until the success, which must be an integer. But, in our case, $r \kappa$ and $\frac{r}{R} \beta$ are not necessarily integer values. Another way for computing the probabilities $\mathbb{P}(a)$ and $\mathbb{P}(b \mid a)$ would be to regard the arrivals of the disclosure packets as a Poisson arrival process, which is the continuous-time equivalent of the Bernoulli. In this case, $X$ would be exponentially distributed with rate $\delta_{r}$. However, if the exponents of the geometric distribution are generally large and the probability $\delta_{r}$ is relatively small (which holds in our case as shown in Fig. 3), then the probabilities obtained using the geometric distribution approximate very well the ones obtained by the exponential distribution. In this work, we have used the geometric distribution, because we believe it provides more intuitive results. A similar analysis holds when considering a Poisson arrival process for the disclosure packets.

Note 2: Lemmas 4.1 and A.1 do not imply any assumption about independence of $F$-arrivals, nor Poisson packet arrivals. The proofs are based on Palm Calculus and linearity of expectation, that do not require independence. The only assumptions here are stationarity and ergodicity. The "steady-rate" or the Poisson assumption make the computation of Eq. 11 easier and provide a good insight of the proof, but do not correspond to real traffic arrival patterns.

Note 3-clarification about the implication of Lemma 4.1: The best prediction for $n_{F}\left[P_{0}, P_{\beta}\right)$ and $n_{F}[0, \kappa)$ is obtained from their expected values. This is because if one wants to predict any random variable from its distribution, then the mean-square-error (MSE) predictor and the best one-number guess is just its expected value. By applying Eqs. 10 and 11 to Eq. 9, we get the best prediction of the sampling probability of an arbitrary packet $p$, as computed at the time of arrival (Palm sampling 
probability). The latter does not depend on p's arrival time, only on the intensities $R$ and $r$. Even if node $i$ knows perfectly $R$ and $r$, when it attempts to predict the sampling probability of each new packet $p$ that it observes, it computes the same sampling probability for all new packets.

\section{A.2 Argument for Claim 4.2}

Our argument consists of an analysis (w.r.t. $t$ and $g$ ) of the expected delay benefit that a misbehaving node $i$ (or, better stated, its domain) has, when launching a prioritization attack. For the analysis, we consider all possible cases for $t>0$ and $g \in(0,1)$ and show that Ineq. 2, 3 are sufficient to make the expected misbehavior benefit negative, i.e. they imply Ineq. 4 :

$$
M B_{d} \equiv \hat{d}_{h o n}-\hat{d}_{m i s}<0
$$

Note that in our context, it is enough to provide attack-resistance guarantees based on expectations. The actual delay benefit cannot be known to any node in advance; it can be computed only at the end of the measurement. So, we can safely assume that if a node will launch a prioritization attack, only if its expected misbehavior benefit is non-negative.

Case a: $t \geq \kappa$. In this misbehavior scenario, node $i$ is able to identify all packets for which disclosure happens inside the quiet period (and are therefore excluded from the sampling process), all packets for which disclosure happens outside the quiet period (out of which samples are picked), and some of the samples.

The exact computation of the expected misbehavior benefit depends on the forwarding strategy that node $i$ follows, but we can commute an upper bound. Since $t \geq \kappa$, the monitor's delay estimate cannot be less than sum of $\kappa$ (which is the minimum buffering time period in this case), and the delay of the good route (which is achieved when the node manages to forward all samples over the good route), i.e.:

$$
\hat{d}_{m i s} \geq d_{g}+\kappa
$$

Therefore,

$$
\begin{aligned}
M B_{d} & \equiv \hat{d}_{\text {hon }}-\hat{d}_{\text {mis }} \\
& =g \cdot d_{g}+(1-g) \cdot d_{b}-\hat{d}_{\text {mis }} \\
& \leq(1-g)\left(d_{b}-d_{g}\right)-\kappa \\
& \stackrel{(2)}{<}-g \Delta_{d} \leq 0,
\end{aligned}
$$

which means that Ineq. 2 implies Ineq. 4; i.e., the condition about $\kappa$ given by Ineq. 2 is sufficient to guarantee that a prioritization attack of Case (a) is unsuccessful.

Case b: $t<\kappa$. For this part, we divide the packets forwarded by a misbehaving node $i$ that launches the prioritization attack into two categories:

- Category I: The packets for which disclosure happens within $t$. Node $i$ knows that these are excluded from the sampling process, because they fall into the quiet period $\kappa$, and it forwards them over the bad route.

- Category II: The packets for which disclosure does not happen within $t$ (hence, each of them is buffered for $t$ before being forwarded). Node $i$ knows that a subset of these will not be excluded, and a subset of the non-excluded ones will be sampled, but it does not know which subsets these are. Hence, it forwards as many as it can over the good route and the rest over the bad route. 
Misbehavior Benefit Function. Let $\Delta_{d}=d_{b}-d_{g}$ be the delay difference of the two routes and $m(t)$ denote the expected fraction of packets for which disclosure happens within $t$ (packets of Category I described above). Let also $t^{\star}$, denote the buffering time at which the bad route is saturated with packets whose disclosure happens during their buffering period and therefore their exclusion for the sampling process is verified by the misbehaving node. I.e.:

$$
m\left(t^{\star}\right)=1-g
$$

Given the attack model that is described in $\S 4.1$, for any $g \in(0,1)$, the expected misbehavior benefit takes the following form w.r.t. $t$ :

$$
M B_{d}= \begin{cases}\frac{g m(t)}{1-m(t)} \Delta_{d}-t & \text { if } 0<t \leq t^{\star} \\ (1-g) \Delta_{d}-t & \text { if } t \geq t^{\star}\end{cases}
$$

Computation of the upper branch of Eq. 14: Due to its definition, $m(t)$ is an increasing function of $t$. Hence:

$$
\begin{aligned}
& m(t) \leq m\left(t^{\star}\right) \\
\stackrel{(17)}{\Longleftrightarrow} & m(t) \leq 1-g \\
\Leftrightarrow & \frac{g}{1-m(t)} \leq 1
\end{aligned}
$$

Ineq. 15 shows that the packets of Category II exceed or exactly match the capacity of the good route. In this case, the node forwards as many of these packets as it can (i.e. $\frac{g}{1-m(t)}$ ) over the good route and the rest (i.e. $1-\frac{g}{1-m(t)}$ ) over the bad route. It does so, because this is the only way it never exceeds the capacities of both routes and it maximizes the number of packets of Category II (from which the sample will be selected) that follow the good route.

Note that the exact algorithm, with which the node manages to perform the above policy at line rates, is not required for this proof. I.e. we consider here the best possible misbehaving scenario that is offered to the node because of the domain's network conditions. This makes our analysis a worst-case approach.

The monitor always produces an accurate delay estimate, based on a sample of packets of Category II. Hence, the expected ${ }^{2}$ value of estimated mean delay of the domain is:

$$
\hat{d}_{m i s}=\frac{g}{1-m(t)}\left(d_{g}+t\right)+\left(1-\frac{g}{1-m(t)}\right)\left(d_{b}+t\right)
$$

Based on Eq. 16, we obtain the upper branch of Eq. 14:

$$
\begin{aligned}
M B_{d}= & g \cdot d_{g}+(1-g) \cdot d_{b}-\hat{d}_{m i s} \\
\stackrel{(16)}{=} & -\frac{g-g m(t)-g}{1-m(t)} d_{g}-t \\
& +\frac{1-g-m(t)+g m(t)-1+g+m(t)}{1-m(t)} d_{b} \\
= & \frac{g m(t)}{1-m(t)} \Delta_{d}-t, \quad \forall t \in\left[0, t^{\star}\right] .
\end{aligned}
$$

\footnotetext{
${ }^{2}$ The exact estimate cannot be known in advance, because it is produced from the collected sample, but the estimate will be very close to the expected value. This is because the number of packets of Category II is smaller than the total flow traffic This, in turn, means that the collected sample (as computed in §D.1) is larger than necessary to estimate the average delay of those packets with adequate accuracy. By fixing $\gamma$ and $\epsilon$ to reasonable values (e.g. $\gamma=95 \%$ and $\epsilon=10 \%$ ), the accuracy of $\hat{d}_{m i s}$ is, almost surely, adequately high.
} 
Computation of the lower branch of Eq. 14: At the buffering time $t=t^{\star}$, the capacity of the bad route is saturated with packets of Category I, and, because of Eq. 17, the misbehavior benefit becomes $M B_{d}\left(t^{\star}\right)=(1-g) \Delta_{d}-t^{\star}$. I.e. all sampled packets follow the good route.

If the node buffers for time $t>t^{\star}$, then the packets will be delayed for more time, while all non-excluded packets will continue to follow the good route. So, $\hat{d}_{m i s}=d_{g}+t$ and thus $M B_{d}=(1-g) \Delta_{d}-t$.

Upper bound for Eq. 14: We can compute an upper bound for the expected misbehavior benefit by finding an upper bound for the expected fraction of packets of Category I, i.e., $m(t)$.

Given a disclosure probability $\delta_{r}$ and a buffering period $t$, for any packet from flow $F$ that arrives at time $t_{0}$, the probability that its disclosure packet arrives within period $t$ follows the geometric distribution with parameter $\delta_{r}$, and it is equal to: $1-\left(1-\delta_{r}\right)^{n_{F}}\left[t_{0}, t_{0}+t\right)$, where $n_{F}\left[t_{0}, t_{0}+t\right)$ is the number of packets from flow $F$ that fall inside the time interval $\left[t_{0}, t_{0}+t\right)$. Therefore, since $n_{F}\left[t_{0}, t_{0}+t\right)$ is random variable, the actual fraction of packets of Category $\mathrm{I}$ is also a random variable.

An upper bound for $m(t)$ can be found using our stationarity assumption and (§2.5) Jensen’s Inequality: Flow $F$ 's packet arrivals form a stationary and ergodic point process with intensity $r$. Therefore, $n_{F}\left[t_{0}, t_{0}+t\right]$ is a random variable whose distribution is invariant under time shifts, i.e., it is the same for any $t_{0}$.

Also, from the definition of intensity $r$ (which is the expected number of points per time unit [5]), the expected value of $n_{F}\left[t_{0}, t_{0}+t\right)$ is: $\mathbb{E}\left[n_{F}\left[t_{0}, t_{0}+t\right)\right]=r t$, for any $t_{0} \geq 0$.

Last, since the function $1-\left(1-\delta_{r}\right)^{n_{F}}\left[t_{0}, t_{0}+t\right)$ is concave w.r.t. $n_{F}\left[t_{0}, t_{0}+t\right)$, from Jensen's Inequality we get:

$$
m(t) \leq 1-\left(1-\delta_{r}\right)^{r t}
$$

Given Ineq. 17, we now obtain an upper bound for the expected misbehavior benefit $M B_{d}$ :

$$
M B_{d} \leq \begin{cases}\frac{g\left(1-(1-\delta)^{r t}\right)}{(1-\delta)^{r t}} \Delta_{d}-t & \text { if } 0<t \leq t^{\star} \\ (1-g) \Delta_{d}-t & \text { if } t \geq t^{\star}\end{cases}
$$

We denote the right hand side of the above with $\max M B_{d}$. I.e.:

$$
\max M B_{d}= \begin{cases}\frac{g\left(1-(1-\delta)^{r t}\right)}{(1-\delta)^{r t}} \Delta_{d}-t & \text { if } 0<t \leq t^{\star} \\ (1-g) \Delta_{d}-t & \text { if } t \geq t^{\star}\end{cases}
$$

Analysis of Eq. 19: The upper branch of Eq. 19 is a convex function of $t$, while the lower branch is a decreasing function of $t$. To see why, we provide hereunder the first and second derivatives of $\max M B_{d}$. One can verify that the second derivative (Eq. 21) of the upper branch is non-negative and the first derivative (Eq. /20) of the lower branch is negative.

$$
\begin{aligned}
& \frac{d\left(\max M B_{d}\right)}{d t}= \begin{cases}g \Delta_{d}\left(-r\left(1-\delta_{r}\right)^{-r t} \ln \left(1-\delta_{r}\right)\right)-1 & , 0<t \leq t^{\star} \\
-1 & , t \geq t^{\star}\end{cases} \\
& \frac{d^{2}\left(\max M B_{d}\right)}{d t^{2}}= \begin{cases}g \Delta_{d}\left(-r \ln \left(1-\delta_{r}\right)\right)^{2}\left(1-\delta_{r}\right)^{-r t} & , 0 \leq t \leq t^{\star} \\
0 & , t \geq t^{\star}\end{cases}
\end{aligned}
$$

Since the maximum misbehavior benefit $\max M B_{d}$ is a continuous function of $t$, that is convex in $\left[0, t^{\star}\right]$ and decreasing for $t \geq t^{\star}$, then, for any $g \in(0,1)$, the function takes its maximum value at one of the edges of the support of its upper branch, i.e. $t=0$ or $t=\min \left\{\kappa, t^{\star}\right\}$ (because of the general assumption of Case b: $t<\kappa)$. At $t=0, M B_{d}(0)=0$, while at $t=t^{\star}$, the sign of $M B_{d}$ depends on the actual values of $g, \delta_{r}$ and $\kappa$. 
Moreover, the maximum misbehavior benefit max $M B_{d}$ is decreasing with $t^{\star}$. We obtain a lower bound for $t^{\star}$ directly from Ineq. 17 and Eq. 13:

$$
t^{\star} \geq \frac{\ln g}{r \ln \left(1-\delta_{r}\right)}
$$

Thus, to show that the conditions of lemma 4.2 about $\kappa$ and $\delta_{r}$ (i.e. Ineqs. 2 and 3 ) imply Ineq. 4 , it is sufficient to show that, when they hold, then:

$$
\begin{aligned}
& \max M B_{d}\left(\min \left\{\kappa, \min \left\{t^{\star}\right\}\right\}, g\right) \\
= & \max M B_{d}\left(\min \left\{\kappa, \frac{\ln g}{r \ln \left(1-\delta_{r}\right)}\right\}, g\right)<0, \quad \forall g \in[0,1]
\end{aligned}
$$

In this case, the expected misbehavior benefit $M B_{d}$ is always non-positive, for any possible $t$ and $g$. We will show this, by considering two sub-cases w.r.t. $g$ :

Case b1: $\left(1-\delta_{r}\right)^{r \kappa} \geq g$. Using this assumption, we get:

$$
\frac{\ln g}{r \ln \left(1-\delta_{r}\right)} \geq \kappa \Rightarrow \min \left\{\kappa, \frac{\ln g}{r \ln \left(1-\delta_{r}\right)}\right\}=\kappa \text {. }
$$

Therefore,

$$
\begin{aligned}
(23) \stackrel{(19),(24)}{\Longleftrightarrow} M B_{d}(\kappa, g) & <(1-g) \Delta_{d}-\kappa \\
& \stackrel{(2)}{<}-g \Delta_{d} \leq 0 .
\end{aligned}
$$

Thus, Ineq. 2 implies Ineq. 23, i.e, the condition about $\kappa$ given by Ineq. 2 is sufficient to guarantee that a prioritization attack of Case (b1) is unsuccessful.

Case b2: $\left(1-\delta_{r}\right)^{r \kappa}<g$. Using this assumption, we get:

$$
\frac{\ln g}{r \ln \left(1-\delta_{r}\right)}<\kappa
$$

Since $\min \left\{t^{\star}\right\}=\frac{\ln g}{r \ln \left(1-\delta_{r}\right)}<\kappa$, no conditions about $\kappa$ exist that provide an upper bound for the maximum possible benefit at $t=\min \left\{t^{\star}\right\}$. The node is able to detect some of the discarded (because of the quiet period) packets, and its best misbehaving strategy is to forward as many as it can over the bad route, which equivalently ensures that all the other packets, among which the samples, will follow the good route. Moreover, at that time, the maximum benefit depends on the values of $g$ and $\delta_{r}$, and it cannot be bounded only through the choice of $\kappa$. It is necessary to properly set $\delta_{r}$, too.

We consider again Ineq. 23 and show why 2 and 3 are sufficient to guarantee the requirement.

$$
\begin{gathered}
(23) \stackrel{(19),(25)}{\Longleftrightarrow}(1-g) \Delta_{d}-\frac{\ln g}{r \ln \left(1-\delta_{r}\right)}<0 \\
\Leftrightarrow \frac{-1}{r \Delta_{d} \ln \left(1-\delta_{r}\right)}>\frac{g-1}{\ln g}
\end{gathered}
$$

First, we need to prove the following auxiliary lemma:

LEMMA A.2. $\ln g \leq g-1$ 
Proof: Take the Taylor expansion of $\ln g$ around $g_{0}=1$ :

$$
\begin{aligned}
\ln g= & \ln g_{0}+\left.\left(g-g_{0}\right) \frac{\mathrm{d} \ln g}{\mathrm{~d} g}\right|_{g=g_{0}} \\
& +\left.\frac{1}{2}\left(g-g_{0}\right)^{2} \frac{\mathrm{d} \ln g}{\mathrm{~d} g^{2}}\right|_{g=\xi}, \text { for some } \xi \in\left[g_{0}, g\right] \\
= & \ln (1)+(g-1) \frac{1}{1}+\frac{1}{2}(g-1)^{2}\left(-\frac{1}{\xi^{2}}\right)^{1}
\end{aligned}
$$

The last term of the above is always non-positive. Thus,

$$
\ln g \leq 0+(g-1)
$$

Because of lemma A.2, we get that $\frac{g-1}{\ln g} \leq 1$.

Now, we go back to Ineq. 26. To show that the inequality is satisfied, it is enough to show that the left hand side (LHS) of the inequality satisfies the following: $L H S$ (Ineq. 26) > 1, for some $\delta_{r} \in(0,1)$. But,

- if $\left(1-\delta_{r}\right)^{r \kappa} \geq \frac{1}{e}$ (i.e. Ineq. 3 holds), then:

$$
\begin{aligned}
& \left(1-\delta_{r}\right)^{r \kappa} \geq \frac{1}{e} \\
\Leftrightarrow \quad & \ln \left(\left(1-\delta_{r}\right)^{r \kappa}\right) \geq-1 \\
\Leftrightarrow & r \kappa \ln \left(1-\delta_{r}\right) \geq-1 \\
\Leftrightarrow & \frac{-1}{r \kappa \ln \left(1-\delta_{r}\right)} \geq 1 .
\end{aligned}
$$

- if additionally $\kappa>\Delta_{d}$ (i.e. Ineq. 2 holds), then:

$$
\begin{aligned}
\frac{1}{\Delta_{d}} & >\frac{1}{\kappa} \\
\Leftrightarrow & \frac{-1}{r \Delta_{d} \ln \left(1-\delta_{r}\right)}>\frac{-1}{r \kappa \ln \left(1-\delta_{r}\right)} \\
\stackrel{(27)}{\Longrightarrow} & \frac{-1}{r \Delta_{d} \ln \left(1-\delta_{r}\right)}>1 \\
\stackrel{(\text { lem.A.2) }}{=} & \frac{-1}{r \Delta_{d} \ln \left(1-\delta_{r}\right)}>\frac{g-1}{\ln g} \rightarrow(26,23) \text { hold }
\end{aligned}
$$

Hence, if an attack is considered unsuccessful according to ineq 4, then Ineq. 2 and 3 are sufficient to guarantee that an attack of Case (b2) is unsuccessful.

In conclusion, by considering both cases (a) and (b), we have showed that for any $t>0$ and any $g \in(0,1)$, Ineq. 2 and 3 are sufficient to make a prioritization attack unsuccessful.

A.2.1 Proof of Inequality 5. The inequality can be derived directly from Eq. 20 and 3:

$$
\begin{gathered}
\frac{d\left(M B_{d}\right)}{d t} \leq \begin{cases}\frac{g}{\left(1-\delta_{r}\right)^{-r t}} \Delta_{d}\left(-r \ln \left(1-\delta_{r}\right)\right)-1 & , t \leq t^{\star} \\
-1 & , t \geq t^{\star}\end{cases} \\
\stackrel{(3)}{\Longrightarrow} \frac{d\left(M B_{d}\right)}{d t} \leq \begin{cases}\frac{g}{\left(1-\delta_{r}\right)^{-r t}} \Delta_{d} \frac{1}{\kappa}-1 & , t \leq t^{\star} \\
-1 & , t \geq t^{\star}\end{cases}
\end{gathered}
$$




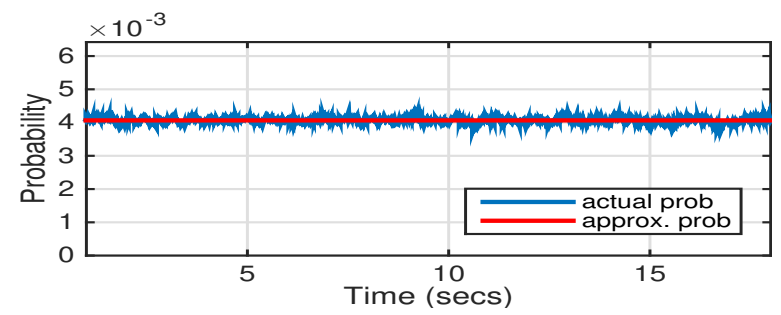

Fig. 9. Palm sampling probability computed at each packet arrival.

And because for $t \leq t^{\star} \stackrel{(22)}{\Longleftarrow} g<\left(1-\delta_{r}\right)^{-r t}$, the above results in:

$$
\begin{aligned}
\frac{d\left(M B_{d}\right)}{d t} & \leq\left\{\begin{array}{cc}
\frac{\Delta_{d}}{\kappa}-1 & , t \leq t^{\star} \\
-1 & , t \geq t^{\star}
\end{array}\right. \\
\stackrel{\frac{\Delta_{d}}{\kappa}>0}{\Longrightarrow} \frac{d\left(M B_{d}\right)}{d t} & \leq \frac{\Delta_{d}}{\kappa}-1
\end{aligned}
$$

\section{A.3 Argument for Claim 5.1}

Let $F$ (resp. $P$ ) denote the stationary and ergodic point process with intensity $r$ (resp. $R_{i}$ ) that corresponds to the packet arrivals from flow $F$ (resp. to the packet arrivals of the total traffic observed by node $i$ ).

Let $1_{\{F(\tau)=\text { sample }\}}$ denote the sampling indicator random variable, i.e., $1_{\{F(\tau)=\text { sample }\}}=1$, if a packet from flow $F$ arrives at the node at time $\tau$ and is sampled, and 0 otherwise. To denote an arbitrary point in time, we will use again the same convention $(\tau=0)$ as in the proof of Lemma 4.1 from Palm Calculus [5] $]^{3}$. That is: $1_{\{F(0)=\text { sample }\}}$ is the indicator random variable of the event "a packet from flow $F$ that arrives at an arbitrary point in time is sampled".

Applying the Palm's inversion formula [5], we get:

$$
\begin{aligned}
\mathbb{E}\left[1_{\{F(\tau)=\text { sample }\}}\right] & =\mathbb{E}\left[1_{\{F(0)=\text { sample }\}}\right] \\
& =r \cdot \mathbb{E}^{0}\left[\int_{0}^{T_{1}} 1_{\{F(s)=\text { sample }\}} d s\right]
\end{aligned}
$$

where $T_{1}$ is the arrival time of the first packet from flow $F$ (or equivalently the occurrence time of the first $F$-point) after $\tau=0$.

By definition, the palm expectation at the right hand side of Eq. 28 is the expected number of samples between two $F$-point occurrences, given that an $F$-point occurs (or equivalently a packet from flow $F$ arrives) at time $\tau=0$, which, in turn, is the palm sampling probability as defined in Eq. 1. I.e.:

$$
\mathbb{E}^{0}\left[\int_{0}^{T_{1}} 1_{\{F(s)=\text { sample }\}} d s\right]=\left(\left(1-\delta_{r}\right)^{n_{F}[0, \kappa)}-\left(1-\delta_{r}\right)^{n_{F}\left[P_{0}, P_{\beta_{i}}\right)}\right) \cdot \sigma
$$

Due to the "fast ergodic convergence" assumption, we can approximate $n_{F}[0, \kappa)$ and $n_{F}\left[P_{0}, P_{\beta_{i}}\right)$ in Eq. 29 with their expected values (as computed in Lemma 4.1). As stated in Section §2.5, each flow $F$ 's packet-arrival rate is high enough (e.g., the rate of a highly utilized OC-12 link or higher) that ergodic convergence is achieved in less than $100 \mathrm{msec}$ (which is the typical value for $\kappa$ ). Therefore, the actual number of $F$-points in an interval larger than or equal to $\kappa$ converges to its expected

\footnotetext{
${ }^{3}$ This convention is the one used to give a meaning to an arbitrary point in time and differs from the beginning of the process; it is the time of arbitrary point in a process that has a stationary regime and has run long enough to be in steady state.
} 
value. Note that the interval $n_{F}\left[P_{0}, P_{\beta_{i}}\right)$ is always larger than or equal to $\kappa$. This is because we are interested in solutions where the buffer size can hold at least $100 \mathrm{msec}$ worth of traffic receipts, otherwise no receipts can be emitted.

More specifically, from Birkhoff's ergodic theorem for stationary processes, we get:

$$
\begin{gathered}
\frac{1}{\kappa} \int_{0}^{\kappa} F(\tau) d \tau \stackrel{\text { a.s. }}{\longrightarrow} \mathbb{E}[F(0)] \\
\frac{1}{\beta_{i}} \sum_{j=1}^{\beta_{i}} F_{P}[j] \stackrel{\text { a.s. }}{\longrightarrow} \mathbb{E}\left[F_{P}[1]\right]
\end{gathered}
$$

Because of the definition of $n_{F}[0, \kappa)$ and $n_{F}\left[P_{0}, P_{\beta_{i}}\right)$, we have the following: $n_{F}[0, \kappa)=\int_{0}^{\kappa} F(\tau) d \tau$ and $n_{F}\left[P_{0}, P_{\beta_{i}}\right)=\sum_{j=1}^{\beta_{i}} F_{P}[j]$. So, $n_{F}[0, \kappa)$ and $n_{F}\left[P_{0}, P_{\beta_{i}}\right)$ converge to $\kappa \cdot \mathbb{E}[F(0)]$ and $\beta_{i} \cdot \mathbb{E}\left[F_{P}[1]\right]$, respectively. Also because of the definition of the intensity $r$ of process $F$ and Lemma A.1, we get: $\mathbb{E}[F(0)]=r$ and $\mathbb{E}\left[F_{P}[1]\right]=\pi_{F}=\frac{r}{R_{i}}$.

To validate the correctness of the latter approximations under real traffic conditions, we conducted a numeric investigation using the real traffic traces made available by CAIDA in 2016 (chicagoequinix, direction A), which we also used for our experimental evaluation (Section §7). For each traffic trace, we computed the "actual" palm sampling probability of all packets in the trace without making the above mentioned approximations. In all cases, we found that approximating $n_{F}[0, \kappa)$ and $n_{F}\left[P_{0}, P_{\beta_{i}}\right)$ with their expected values worked very well in practice. Fig. 9 depicts an example case of a 20-sec trace (Equinix-chicago April 2016). The actual and the approximated probabilities for all packets are indeed very close.

Thus, Eq. 28 becomes:

$$
\mathbb{E}\left[1_{\{F(\tau)=\text { sample }\}}\right] \approx r \cdot\left(\left(1-\delta_{r}\right)^{r \cdot \kappa}-\left(1-\delta_{r}\right)^{\frac{r}{R_{i}} \cdot \beta_{i}}\right) \cdot \sigma,
$$

which is an approximation of the probability of the event "a packet from flow $F$ arrives at the node at an arbitrary point of time $\tau=0$ and is sampled".

Using the linearity property of expectation, we conclude that the expected number of sampled packets (or equivalently, the expected number of receipts that a node emits) w.r.t to flow $F$ within a random time interval $T$, is approximated by:

$$
\begin{aligned}
\mathbb{E}\left[\int_{0}^{T} 1_{\{F(\tau)=\text { sample }\}} d \tau\right] & =\int_{0}^{T} \mathbb{E}\left[1_{\{F(\tau)=\text { sample }\}}\right] d \tau \\
& \approx T \cdot r \cdot\left(\left(1-\delta_{r}\right)^{r \cdot \kappa}-\left(1-\delta_{r}\right)^{\frac{r}{R_{i}} \cdot \beta_{i}}\right) \cdot \sigma
\end{aligned}
$$

\section{A.4 Argument for Claim 5.2}

We are considering a flow $F$ that traverses a node $i$ and then another node $o$.

Let $F$ denote the stationary and ergodic process of $F$ 's packet arrivals at $i$, which has intensity $r$.

Let $P_{i}$ (resp. $P_{o}$ ) denote the stationary and ergodic process of all packet arrivals at $i$ (resp.o), which has intensity $R_{i}$ (resp. $R_{o}$ ).

Node $i$ (resp. $o$ ) has buffer size $\beta_{i}$ (resp. $\beta_{o}$ ).

Consider a packet $p$ from $F$ that arrives at $i$ at an arbitrary point in time $\tau=0$, when $F, P_{i}$, and $P_{o}$ have run long enough to be in steady state, then arrives at node $o$ at a later point in time. Suppose $i$ picks $d$ as $p$ 's disclosure packet, $p$ does not suffer early or late disclosure at $i$, and $i$ samples $p$. As stated in $\S 3.5$, node $o$ will also sample $p$, except for the four cases stated in that section.

Condition I of Claim 5.2 eliminates Cases I(a) and I(b). 
Condition II eliminates Case II: Suppose $d$ arrives at node $i$ (resp. o) $\Delta_{i}$ (resp. $\Delta_{o}$ ) time units after $p$. Given that $p$ does not suffer early disclosure at $i, \Delta_{i}>\kappa$. Given that the jitter between $i$ and $o$ is below $\mu, \Delta_{o}>\Delta_{i}-\mu$. Hence, according to Alg. 1 (lines 12,13), o samples $p$.

We now show that Condition III $\left(\frac{\beta_{i}}{R_{i}}=\frac{\beta_{o}}{R_{o}}=\frac{\beta}{R}\right)$ and our "fast ergodic convergence" assumption (§2.5) eliminate Case III:

Let $n_{F}\left[F_{0}, F_{d}\right)$ denote the number of $F$-points between $p$ 's and $d$ 's arrivals.

Let $n_{P_{i}}\left[F_{0}, F_{d}\right)$ denote the number of $P_{i}$-points between $p$ 's and $d$ 's arrivals at node $i$.

Let $n_{P_{o}}\left[F_{0}, F_{d}\right)$ denote the number of $P_{o}$-points between $p$ 's and $d$ 's arrivals at node $o$.

Because of Eq. 12:

$$
\begin{aligned}
\mathbb{E}\left[n_{P_{i}}\left[F_{0}, F_{d}\right)\right] & =\frac{R_{i}}{r} \cdot n_{F}\left[F_{0}, F_{d}\right) . \\
\mathbb{E}\left[n_{P_{o}}\left[F_{0}, F_{d}\right)\right] & =\frac{R_{o}}{r} \cdot n_{F}\left[F_{0}, F_{d}\right) .
\end{aligned}
$$

Because of the "fast ergodic convergence" assumption (§2.5) and Birkhoff's ergodic theorem for stationary processes, $n_{P_{i}}\left[F_{0}, F_{d}\right)$ and $n_{P_{o}}\left[F_{0}, F_{d}\right)$ converge to their expected value almost surely (a.s.).

The fact that $i$ samples $p$ implies that:

$$
n_{P_{i}}\left[F_{0}, F_{d}\right) \leq \beta_{i}
$$

Combining the above statements with Condition III $\left(\frac{\beta_{i}}{R_{i}}=\frac{\beta_{o}}{R_{o}}\right)$, we have:

$$
\begin{aligned}
n_{P_{i}}\left[F_{0}, F_{d}\right) & \leq \beta_{i} \\
& \stackrel{\text { a.s. }}{\Longrightarrow} \mathbb{E}\left[n_{P_{i}}\left[F_{0}, F_{d}\right)\right] \leq \beta_{i} \\
& \Leftrightarrow \frac{R_{i}}{r} \cdot n_{F}\left[F_{0}, F_{d}\right) \leq \beta_{i} \\
& \Leftrightarrow \frac{R_{o}}{r} \cdot n_{F}\left[F_{0}, F_{d}\right) \leq \beta_{o} \\
& \Leftrightarrow \mathbb{E}\left[n_{P_{o}}\left[F_{0}, F_{d}\right)\right] \leq \beta_{o} \\
& \stackrel{a . s .}{\Longrightarrow} n_{P_{o}}\left[F_{0}, F_{d}\right) \leq \beta_{o} .
\end{aligned}
$$

This implies that $p$ does not suffer late disclosure at $o$.

\section{B OTHER MISBEHAVIOR}

Fake late disclosure. If domain $x$ does not perform well w.r.t. flow $F, x$ may try to prevent the monitor from estimating $x$ 's performance w.r.t. $F$, by emitting fake late-disclosure warnings. This does not help $x$, because a fake late-disclosure warning has the same effect as a fake-receipt attack (§2.4): the best a domain can do is shift the blame for some loss/delay to one of its own inter-domain links. For instance, suppose flow $F$ crosses three subsequent nodes $i_{x}, o_{x}$ (in domain $x$ ), and $i_{y}$ (in domain $y$ ). Suppose $o_{x}$ emits a late-disclosure warning. As a result, for a short time interval, the monitor cannot estimate the performance of the intra-domain segment $\left\{i_{x}, o_{x}\right\}$ or the inter-domain link $\left\{o_{x}, i_{y}\right\}$. However, the monitor can still estimate the performance of the segment $\left\{i_{x}, i_{y}\right\}$. Hence, $o_{x}$ 's warning does not prevent the monitor from correctly estimating $x$ 's loss/delay, it only makes it unclear whether this loss/delay occurred on the intra-domain segment or the inter-domain link adjacent to $o_{x}$.

Misconfiguration. We showed that a prioritization attack always harms the misbehaving domain's perceived delay performance, as long as $\kappa$ and $\delta_{r}$ are set according to Claim 4.2. Domain $x$ may purposefully set $\kappa$ and $\delta_{r}$ such that the conditions of Claim 4.2 do not hold. This does not help $x$ : Each participating node's parameters must be set according to well-defined rules, which 
include honoring the inequalities of Claim 4.2 (§6.1); if a node's parameters are set incorrectly, this is apparent from the receipts it emits. For instance, a node's quiet-period duration $\kappa$ is apparent to the monitor, because the node does not emit receipts during quiet periods; if a node uses a shorter $\kappa$ than it should, it will emit receipts when it should not (too close to the corresponding disclosure packets), and the monitor will immediately detect that and ignore the node's receipts. Hence, emitting receipts produced with incorrect parameters is equivalent to emitting no receipts.

Forceful disclosure. A source that generates a flow $F$ of packet rate $r$ may purposefully craft packets that will be picked as disclosure packets, in order to cause the nodes that observe $F$ to process it with a particular disclosure rate that is different from the proper disclosure rate $\delta_{r}$ set in each node according to Claim 4.2. The result is the same as if the nodes that observe $F$ are misconfigured, i.e., they have set $\delta_{r}$ incorrectly. However, it is important to note that this behavior affects only the disclosure process for the particular flow $F$ generated by the misbehaving source (because one flow's traffic cannot affect another flow's disclosure process).

\section{SAMPLING INCONSISTENCY}

In general, inconsistent sampling does not lead to incorrect performance estimates, because the monitor detects the inconsistency and discards the corresponding samples. Inconsistent sampling simply means that the monitor must wait longer to collect the minimum sample size necessary to achieve the target accuracy, because some of the samples are discarded.

In §3.5, we stated all the cases where nodes may sample inconsistently. We can eliminate Case III through proper parametrization (Claim 5.2, Condition III). We now discuss Cases I(a) and I(b) (Case II is straightforward).

Consider a flow $F$ that traverses nodes $i$ and $o$.

Case I(a): Consider a packet sequence $\left\{d_{0}, \ldots p, \ldots d_{1}, \ldots d_{2}\right\}$ from $F$ and suppose that $d_{1}$ is dropped between $i$ and $o$; as a result, $i$ picks $d_{1}$ as $p$ 's disclosure packet, whereas $o$ picks $d_{2}$ as $p$ 's disclosure packet, which may result in a different sampling outcome for $p$.

The monitor detects that nodes $i$ and $o$ picked different disclosure packets for $p$, because $i$ emits a receipt for $d_{1}$ whereas $o$ does not. To avoid drawing incorrect conclusions, the monitor does not estimate the performance of the $i$-o segment with respect to $F$ between packets $d_{0}$ and $d_{1}$.

Case I $\mathbf{I}(\mathbf{b})$ : Consider a packet sequence $\left\{d_{1}, p, \ldots d_{2}\right\}$ from $F$ and suppose that $d_{1}$ is reordered with $p$ between $i$ and $o$; as a result, node $i$ picks $d_{2}$ as $p$ 's disclosure packet, whereas node $o$ picks $d_{1}$ as $p$ 's disclosure packet, which may result in a different sampling outcome for $p$.

When there exists a reordering element between nodes $i$ and $o$, there will be some packets sampled by $i$ and not by $o$, but also some packets sampled by $o$ and not by $i$. The former could be mistaken for loss between the two nodes, however, the latter indicates packet reordering between them. Hence, the monitor does not estimate the performance of the $i$-o segment with respect to $F$ between packets $d_{0}$ and $d_{1}$. There exist more sophisticated ways to deal with packet reordering, but we think that it does not make sense to complicate the mechanism given that, these days, reordering events are the exception rather than the rule.

\section{COMPUTATIONS}

\section{D.1 Minimum Sample Size}

As stated in $\S 3.5$, the monitor computes the minimum sample size $N_{\gamma, \epsilon}$ necessary to achieve the target $(\gamma, \epsilon)$-accuracy using standard statistics. We now describe how.

\section{Estimation of packet loss.}


In general, $N_{\gamma, \epsilon}$ is a function of the target accuracy $(\epsilon$ and $\gamma$ ) and some distribution moment of the quantity we want to estimate (packet loss, in our case). Since we do not know in advance the distribution of packet loss, we make one of two typical assumptions:

(1) Packet loss is a Bernoulli process: given the sequence of packets from flow $F$ that arrive at node $i$, any packet is lost before reaching node $o$ with probability $l \geq \operatorname{loss}_{\min }$, and packet-loss events are independent. In this case, the standard central limit theorem (CLT) yields a closed formula for $N_{\gamma, \epsilon}$ :

$$
N_{\gamma, \epsilon}=\left(\frac{\eta}{\epsilon}\right)^{2} \cdot \frac{1-\text { loss }_{\min }}{\text { loss }_{\min }}
$$

where $\eta$ is the $\frac{1+\gamma}{2}$-th quantile of the standard normal distribution.

(2) Packet loss is a bursty process governed by the Gilbert model [16]: it is either in a "low-loss" (good) state or in a "high-loss" (bad) state, and it transitions between the two with given probabilities. In this case, we approximate $N_{\gamma, \epsilon}$ empirically through Monte-Carlo simulations.

We can choose one of these two assumptions based on the nature of the measured traffic (how bursty we expect it to be). If we want to be conservative, we must choose (2), which yields a sufficient sample size for both bursty and non-bursty traffic. The disadvantage is that, in the non-bursty case, the resulting sample size will be larger than necessary, i.e., we will use more resources than necessary to meet our target accuracy.

\section{Estimation of delay quantiles.}

The minimum $N_{\gamma, \epsilon}$ necessary to estimate the $\chi$-quantile of the delay distribution with $(\gamma, \epsilon)$ accuracy is given by the theorem of the " $\gamma$-confidence interval for the sample median and other quantiles" $[5,22]$ :

$$
N_{\gamma, \epsilon}=\left(\frac{\eta}{\epsilon}\right)^{2} \cdot \frac{1-\chi}{\chi}
$$

where $\eta$ is the $\frac{1+\gamma}{2}$-th quantile of the standard normal distribution and $0<\chi<1$. Note that, in the context of quantile estimation, the error $\epsilon$ is defined as the maximum difference, not between the actual quantile and the estimated one-as one might expect-but between the index of the actual quantile and the index of the estimated one at the order statistic. For example, suppose we want to estimate the 75-th percentile of the delay distribution with $(\gamma, \epsilon)$-accuracy, then Eq. 32 gives the necessary sample size $N_{\gamma, \epsilon}$, which guarantees that the actual delay quantile exists in the interval between the $(0.75 \cdot(1-\epsilon) \cdot N)$-th and $(0.75 \cdot(1+\epsilon) \cdot N)$-th order statistics of the sample, with probability $\gamma$. This is because of the normal approximation of the binomial distribution that is proposed by the theorem for large sample sizes $N_{\gamma, \epsilon}$ [5].

According to Equations 31 and 32, assuming loss $\min \ll 50 \%$, the minimum sample size $N_{\gamma, \epsilon}$ necessary for estimating the $\chi$-quantile of the delay distribution for $\chi \geq 50 \%$ is less than the $N_{\gamma, \epsilon}$ necessary for estimating packet loss with the same accuracy. Hence, we pick $N_{\gamma, \epsilon}$ based on Equation 31.

\section{D.2 Minimum Buffer Size}

As stated in $\S 6.1$, we compute the minimum value of $\frac{\beta}{R}$ for which both Inequalities 3 and 6 are satisfied and set each node's buffer size $\beta$ accordingly. We now describe how.

Recall that, at this point, we have set all parameters except for the nodes' buffer sizes and the disclosure function $r \rightarrow \delta_{r}$. 
We start from Inequality 6 and solve for $\frac{\beta}{R}$ :

$$
\frac{\beta}{R} \geq \frac{\ln \left(\left(1-\delta_{r}\right)^{r \kappa}-\frac{N_{\gamma, \epsilon}}{r \cdot T \cdot \sigma}\right)}{\ln \left(1-\delta_{r}\right)^{r}} .
$$

We want Inequality 33 to hold for any flow intensity $r \in\left[r_{\min }, r_{\max }\right]$. We observe that the term on the right is a monotonically decreasing function of $r$. Hence, if we set $\frac{\beta}{R}$ such that the inequality holds for $r=r_{\text {min }}$, it will hold for any $r \in\left[r_{\min }, r_{\max }\right]$ :

$$
\frac{\beta}{R} \geq \frac{\ln \left(\left(1-\delta_{r}\right)^{r_{\min } \kappa}-\frac{N_{Y, \epsilon}}{r_{\min } \cdot T \cdot \sigma}\right)}{\ln \left(1-\delta_{r}\right)^{r_{\min }}} .
$$

We want the minimum value of $\frac{\beta}{R}$ for which both Inequality 3 and Inequality 34 hold:

- We constrain $\delta_{r}$ according to Inequality 3:

$$
\delta_{r} \in\left(0,1-e^{-\frac{1}{r \kappa}}\right) .
$$

- We observe that the term on the right of Inequality 34 is a convex function of $\delta_{r}$. Hence, it is minimized for some value of $\delta_{r}=\delta_{r}^{*} \in\left(0,1-e^{-\frac{1}{r \kappa}}\right)$ that we compute numerically:

$$
\min _{\delta_{r}}\left\{\frac{\beta}{R}\right\}=\frac{\ln \left(\left(1-\delta_{r}^{*}\right)^{r_{\min } \kappa}-\frac{N_{\gamma, \epsilon}}{r_{\min } \cdot T \cdot \sigma}\right)}{\ln \left(1-\delta_{r}^{*}\right)^{r_{\min }}} .
$$

Equation 35 gives the minimum value of $\frac{\beta}{R}$ for which both Inequalities 3 and 6 hold. Hence, if a node observes packet arrivals of maximum intensity $R^{*}$, we set its buffer size to

$$
\beta^{*}=R^{*} \min _{\delta_{r}}\left\{\frac{\beta}{R}\right\} .
$$

So, in general, each node has a different buffer size, but the ratio $\frac{\beta}{R}$ is the same in all nodes. This also means that the expected fraction of packets that suffer late disclosure is the same in all nodes.

Fig. 10 shows a node's minimum buffer size in various realistic scenarios, as a function of the minimum supported flow intensity $r_{\min }(10 \mathrm{a})$, the measurement interval $T(10 \mathrm{~b})$, and the minimum loss rate loss $_{\min }(10 \mathrm{c})$. For instance, assume $r_{\min }=155 \mathrm{~K}$ packets $/ \mathrm{sec}$, which corresponds to a saturated OC- 12 link assuming an average packet size $=500$ bytes. Fig. $10 \mathrm{~b}$ shows that a few MB of memory are sufficient for measuring loss $_{\min }=0.1 \%$ in $T=10$ minutes. The plots were computed assuming: $\kappa=100 \mathrm{msec}, \epsilon=10 \%, \gamma=95 \%, R=2.5 \mathrm{Mpkts} / \mathrm{sec}$ (saturated OC-192 link assuming an average packet size $=500$ bytes), and a receipt size of 12 bytes.

We see that the buffer size drops rapidly as $r_{\min }, T$, or loss $_{\min }$ increase, which makes sense: In general, the larger a node's buffer, the less often packets suffer late disclosure at the node, hence, the more samples the node collects per time unit. Higher loss $_{\min }$ means that the monitor needs fewer samples to reach a given target accuracy, hence each node needs to collect fewer samples per time unit. Higher $T$ means that the monitor is willing to wait longer to reach a given target accuracy, hence, again, each node needs to collect fewer samples per time unit. Higher $r_{\min }$ means that we are measuring bigger flows, which contribute more packets per time unit, hence the inter-arrival time between disclosure packets is shorter for a given disclosure rate; as a result, each node needs a smaller buffer to avoid late disclosure with a given probability and collect a given number of samples per time unit.

Clarification: In Figs. 10b and 10c, we see that there always exists a minimum $T$ and a minimum loss $_{\min }$ at which $\beta^{*}$ becomes infinite, i.e., at these points, we could not achieve the target accuracy 


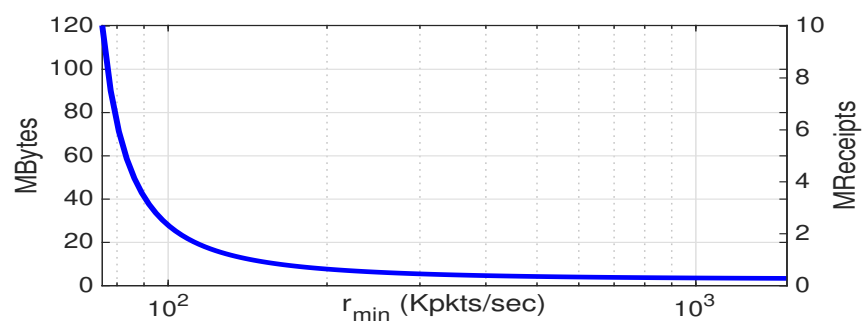

(a) $\beta^{*}$ as a function of $r_{\text {min }}$. loss $_{\min }=0.1 \%, \sigma=1 \%, T=10 \mathrm{~min}$.

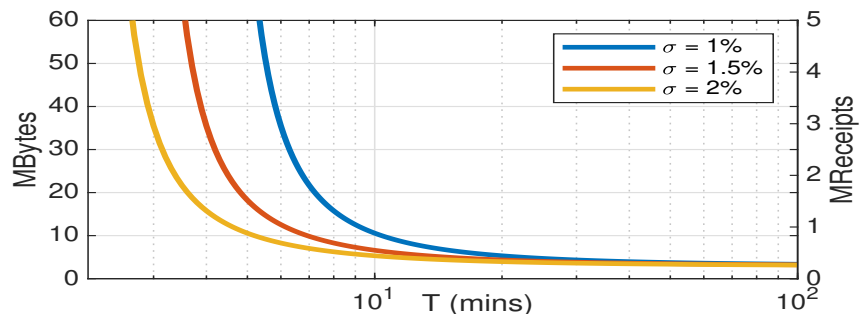

(b) $\beta^{*}$ as a function of $T$. loss $_{\min }=0.1 \%, r_{\text {min }}=155 \mathrm{~K}$ packets $/ \mathrm{sec}$.

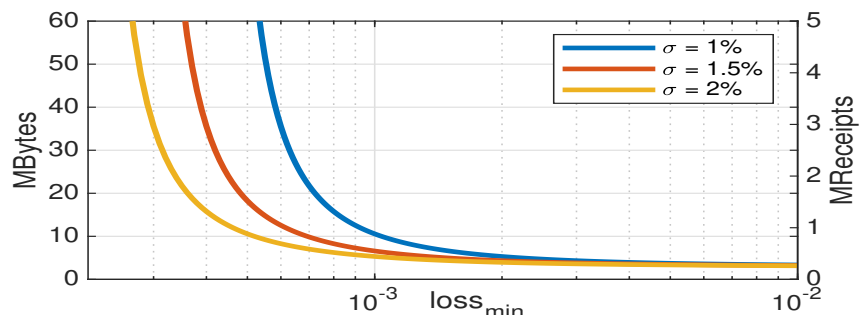

(c) $\beta^{*}$ as a function of loss $\min$.

$T=10 \mathrm{~min}, r_{\min }=155 \mathrm{~K}$ packets $/ \mathrm{sec}$.

Fig. 10. Minimum buffer size $\beta$ in various scenarios.

even with an infinite buffer size. This is because, at these points, the number of packets contributed by the smallest flows in our supported range falls below the minimum necessary sample size.

\section{D.3 Disclosure Function}

As stated in $\S 6.1$, we set our disclosure function such that any tuple $\left\{\delta_{r}, r\right\}$ falls in the operational regime. We now describe how.

First, we compute the operational regime:

- We analytically compute all the tuples $\left\{\delta_{r}, r\right\}$ that satisfy Inequality 3 : solving the inequality for $r$ yields an upper bound for $r$ as a function of $\delta_{r}$ (the middle, yellow curve in Fig. 3).

- We numerically ${ }^{4}$ compute all the tuples $\left\{\delta_{r}, r\right\}$ that satisfy Inequality 6: solving the inequality for $r$ yields an upper and a lower bound for $r$ as a function of $\delta_{r}$ (top/red curve and bottom/blue curve in Fig. 3).

\footnotetext{
${ }^{4}$ We can only do this numerically. Solving Ineq. 6 for $r$ analytically would require solving a polynomial of degree $\frac{\beta}{R}-\kappa$.
} 
The space enclosed between the lower bound and the smaller of the two upper bounds is the operational regime, and our disclosure function must be in that space.

Second, we divide $\left[r_{\min }, r_{\max }\right]$ into non-overlapping packet-rate "zones" and map each zone to a disclosure rate using as few zones as possible (two zones in Fig. 3).

The disclosure function affects the implementation of our algorithm in the following sense: The algorithm tracks each flow's packet rate $r$ in order to choose disclosure packets from that flow with the right probability $\delta_{r}$ (lines 3,4 in Alg. 1). The fewer disclosure rates we use, the less precise our packet-rate tracking needs to be, which simplifies our algorithm's implementation. For example, if we use only two disclosure rates as in Fig. 3, then our packet-rate tracking needs to be only precise enough to determine whether a flow's packet rate is below or above $437 \mathrm{Kpps}$. We found 2 or 3 disclosure rates to be enough in all the scenarios we considered.

\section{E IMPLEMENTATION}

\section{E.1 Software Implementation}

Our algorithm would typically be implemented as part of a hardware data-plane (otherwise we would not need to worry about using expensive data-path memory); still, we implemented a software prototype, to make the point that, despite the complicated analysis, the algorithm itself is straightforward to implement. We used the Data Plane Development Kit [12] on a commodity server equipped with an Intel Xeon E5-2680 CPU and a 10GigE Network Interface Card (NIC) To implement the necessary hash functions, we used a Message Authentication Code (MAC) based on the AES block cipher, leveraging Intel AES-NI and the CPU's hardware support for AES operations [15].

We implemented Alg. 1 in two parallel threads, each running on its own separate core: a "producer" thread that processes incoming packets, computes receipts, and adds them to one end of the receipt buffer; and a "consumer" thread that removes receipts from the other end of the receipt buffer, identifies the right disclosure packet for each receipt, and determines whether to emit or discard the receipt. A receipt consists of a digest, a timestamp, and a flow ID (§2.3). We reserve 4B for the digest, which is computed over the first $48 \mathrm{~B}$ of the packet, modulo the mutable IP header fields (DiffServ, ECN, TTL, and checksum); we confirmed, based on CAIDA traces, earlier results that using $48 \mathrm{~B}$ of the packet's immutable content drops the fraction of non-unique digests to $10^{-3}$ [13]. We reserve $2 \mathrm{~B}$ for the timestamp, which has a granularity of $1 \mathrm{msec}$. And we reserve $6 \mathrm{~B}$ for the flow ID, which is a concatenation of the packet's / 24 IP source and destination prefixes. In total, each receipt requires $12 \mathrm{~B}$.

We ran a simple experiment where a traffic generator sends fixed-size packets to the node, which then forwards them to a sink; we used Spirent SPT-N4U-220 as both traffic generator and sink; we varied packet size from 64 to 1518 bytes. We compared the throughput achieved by our node to the one achieved by a node that performs simple forwarding (without any packet processing). For packet sizes of 128 and higher, both systems forward at the maximum possible packet rate without performance impairment. For 64-byte packets, our node is CPU bottlenecked; the simple forwarder's throughput is $14.88 \mathrm{Mpps}$, while our node's throughput is $14.14 \mathrm{Mpps}-\mathrm{a} \%$ degradation, which indicates that our algorithm introduces on average $1.35 \mathrm{usec}$ extra processing time per packet in this particular platform.

\section{E.2 Sketch of a Hardware Implementation}

Like our software implementation (§E.1), our proposed hardware implementation consists of two parallel threads: a "producer" thread that processes incoming packets, computes receipts, and adds them to the local state; and a "consumer" thread that removes receipts from the local state 
and determines whether to emit or discard each receipt. The local state, however, is organized differently: there is a receipt buffer for "normal" packets, stored in SRAM and accessible only as a queue; and a receipt buffer for disclosure packets, stored in content-addressable memory (CAM) that supports $O(1)$ parallel lookups on the flowID field.

Organizing state in separate receipt buffers for normal and disclosure packets (as opposed to a common receipt buffer) does not introduce any extra or any per-flow state; we keep exactly as many receipts for normal and disclosure packets as Alg. 1, we just store them in two different kinds of memory, because receipts for disclosure packets need to be looked up by flowID (hence require $\mathrm{CAM}$ ), whereas receipts for normal packets, which constitute the vast majority, do not (hence can be stored in cheaper SRAM). For instance, consider the disclosure rates in the example of Fig. 3 : the higher of the two is $2.18 \cdot 10^{-5} \%$, which means that up to this fraction of observed packets are picked as disclosure packets, whereas up to $\sigma=1 \%$ of observed packets in total are sampled. So, if our analysis calls for a receipt buffer of $10.5 \mathrm{MB}$ (as in the particular example), about $0.23 \mathrm{~KB}$ of these need to be CAM, while the rest can be SRAM.

The consumer thread performs the actual sampling: It iterates over the receipt buffer for normal packets, removes receipts, and performs the following operations for each receipt $R$ that corresponds to a normal packet $p$ : (a) It retrieves the set of all receipts $\mathcal{R}$ in the disclosure buffer with the same flowID as $p$. (b) It finds in $\mathcal{R}$ the receipt $R^{\prime}$ that corresponds to $p$ 's disclosure packet: it is the one with the earliest timestamp that satisfies $R^{\prime}$.time $>R$.time. It increments $R^{\prime}$.packetCtr. It determines whether to exclude or select $p$ according to lines 10 and 12 of Alg. 1. (c) It checks whether disclosure may have occurred late for $p$, i.e., if there is no receipt in $\mathcal{R}$ with time $<R$.time. If so, it emits a warning.

The producer thread creates the local state: It processes each incoming packet $p$, computes a receipt $R^{\prime}$ for it, extracts the flowID, retrieves $F$ 's packet rate, checks whether to pick $p$ as a disclosure packet according to line 4 of Alg. 1 , and adds $R^{\prime}$ to the appropriate receipt buffer.

Moreover, the producer thread tracks flow packet rates based on information that is piggy-backed by the consumer thread on the disclosure receipts. We previously said that receipts consist of four fields (§2.3); receipts for disclosure packets have two additional fields for packet-rate tracking, packetRate and packetCtr. Consider a flow $F$ and suppose the first disclosure packet $d_{1}$ arrives at time $t_{1}$; when the second disclosure packet $d_{2}$ arrives at time $t_{2}$, the producer thread retrieves $d_{1}$ 's receipt $R$, estimates $F$ 's packet rate (as $R$.packetCtr divided by $t_{2}-t_{1}$ ), and stores the estimate in $d_{2}$ 's receipt (in the packetRate field); when the third disclosure packet $d_{3}$ arrives, the node repeats the process, computes a new packet-rate estimate, and stores it in $d_{3}$ 's receipt; and so on. The node may compute a brand new estimate every time a disclosure packet arrives, or it may update the previous estimate, akin to keeping a moving-average filter [17]; we choose the latter, because it ensures a smooth variation of estimate without risking sharp increases in rare cases where disclosure packets arrive very close to each other.

We do not need perfect packet-rate tracking: Suppose a flow's real packet rate is $r$, while the estimated one is $\hat{r}$. If $r$ and $\hat{r}$ are in the same zone (map to the same disclosure rate), the disclosure process works as intended; if they map to different disclosure rates, then, of course, it is possible that the chosen disclosure rate is too high or too low for the real packet rate, and we miss the desired accuracy until the estimate catches up with the real value. The fewer disclosure-rate values we use, the less likely we are to hit this scenario, and this is why we designed our disclosure process to operate with smallest number of disclosure-rate values.

With this design, each node performs per packet: two hash computations, a couple of timestamp comparisons, a read and a write access to SRAM, and a parallel lookup and an update in the CAM.

Received August 2018; revised December 2018; accepted January 2019 